\title{
Repetitive ischemic injuries to the kidneys result in lymph node fibrosis and impaired healing
}

Omar H. Maarouf,, ${ }^{1,2}$ Mayuko Uehara, ${ }^{1}$ Vivek Kasinath, ${ }^{1}$ Zhabiz Solhjou, ${ }^{1}$ Naima Banouni, ${ }^{1}$

Baharak Bahmani, ${ }^{1}$ Liwei Jiang, ${ }^{1}$ Osman A. Yilmam, ${ }^{1}$ Indira Guleria, ${ }^{1}$ Scott B. Lovitch, ${ }^{3}$

Jane L. Grogan, ${ }^{4}$ Paolo Fiorina, ${ }^{5}$ Peter T. Sage, ${ }^{1}$ Jonathan S. Bromberg, ${ }^{6}$ Martina M. McGrath, ${ }^{1}$ and Reza Abdi'

'Transplantation Research Center, Renal Division, Brigham and Women's Hospital, Harvard Medical School, Boston,

Massachusetts, USA. ${ }^{2}$ Renal Division, Thomas Jefferson University Hospital, Philadelphia, Pennsylvania, USA.

3Department of Pathology, Brigham and Women's Hospital, Harvard Medical School, Boston, Massachusetts, USA.

${ }^{4}$ Department of Cancer Immunology, Genentech, Inc., South San Francisco, California, USA. ${ }^{5}$ Nephrology Division, Boston

Children's Hospital, Harvard Medical School, Boston, Massachusetts, USA. ${ }^{6}$ Department of Surgery, University of Maryland

School of Medicine, Baltimore, Maryland, USA.

The contribution of the kidney-draining lymph node (KLN) to the pathogenesis of ischemiareperfusion injury (IRI) of the kidney and its subsequent recovery has not been explored in depth. In addition, the mechanism by which repetitive IRI contributes to renal fibrosis remains poorly understood. Herein, we have found that IRI of the kidney is associated with expansion of high endothelial venules (HEVs) and activation of fibroblastic reticular cells (FRCs) in the KLN, as demonstrated by significant expansion in the extracellular matrix. The lymphotoxin $\alpha$ signaling pathway mediates activation of FRCs, and chronic treatment with lymphotoxin $\beta$ receptor-immunoglobulin fusion protein $(\mathrm{LT} \beta \mathrm{r}-\mathrm{Ig})$ resulted in marked alteration of the KLN as well as augmentation of renal fibrosis. Depletion of FRCs reduced T cell activation in the KLN and ameliorated renal injury in acute IRI. Repetitive renal IRI was associated with senescence of FRCs, fibrosis of the KLN, and renal scarring, which were ameliorated by FRC administration. Therefore, our study emphasizes the critical role of FRCs in both the initiation and repair phases of injury following IRI of the kidney.

Authorship note: $\mathrm{OHM}$ and MU contributed equally to this work as co-first authors.

Conflict of interest: The authors have declared that no conflict of interest exists.

Submitted: February 14, 2018

Accepted: May 23, 2018

Published: July 12, 2018

Reference information: JCI Insight. 2018;3(13):e120546. https://doi.org/10.1172/jici. insight.120546.

\section{Introduction}

Acute kidney injury (AKI) affects $4 \%-20 \%$ of hospitalized patients in the US $(1,2)$, including up to $50 \%$ of patients in the intensive care unit (3), and it is associated with a mortality rate approaching 60\% (4-6). Moreover, $\mathrm{AKI}$ is a predictive factor for end-stage renal disease (ESRD), especially among patients who experience multiple ischemic injuries (7). Ischemia-reperfusion injury (IRI) is the culprit behind nearly half of the cases of AKI (8). However, experimental studies exploring the roles of innate and adaptive immunity in ischemic AKI have focused mostly on the kidney itself (9).

The lymph node (LN) is a critical site for immune activation, and it is central to the pathogenesis of antigen-specific immunity (10). The function of the LN is dependent on its specific microvascular, microarchitectural, and cellular compartments, which together support the processes of antigen recognition and recruitment of immune cells from the circulation (11). During normal immune surveillance, naive $\mathrm{T}$ cells home to the LN via high endothelial venules (HEVs) several times a day, surveying for the presence of antigens (12). HEVs are located in the paracortical T cell zone of the LN and surrounded by layers of fibroblastic reticular cells (FRCs), which form 3-dimensional conduits through which T cells can migrate (13-15).

FRCs, identified both by the expression of podoplanin (PDPN) and the absence of endothelial and leukocyte markers, are fibroblast-like cells of mesodermal origin that reside in the interstitium of the LN. FRCs play a critical role in the trafficking of naive T cells into the LN by secreting chemokines, such as CCL19 and CCL21 (16-21). In addition, FRCs produce a highly organized interconnected framework of extracellular matrix (ECM) fibers, generating conduits for the movement of antigens and immune cells within the LN (11, 
13). FRCs also maintain the integrity of HEVs (22). Finally, FRCs contribute to the recovery of the LN following antigenic stimulation (23). In the setting of persistent infection, FRCs accumulate permanent damage, marked by the production of excessive amounts of collagen, leading to fibrosis of the LN (24-26).

The role of the kidney-draining LN (KLN) in the pathogenesis of IRI and its subsequent healing phase is relatively unknown, as is the impact of IRI on KLN structure. The importance of innate and adaptive immune responses to the pathogenesis of renal IRI has been investigated (27), but the majority of these studies have focused on the recruitment and activation status of immune cells within the kidney, and the KLN has received less attention.

The objective of this study is to investigate the impact of IRI on FRCs and key elements of the ECM in the KLN. We demonstrate that the KLN is a critical site of T cell activation following IRI. The KLN undergoes major structural changes in the acute phase following IRI, driven by the activation of FRCs. However, repetitive IRI (IRI ${ }^{\text {(rep) }}$ ) results in chronic changes in the microarchitecture of the KLN, including the accumulation of collagen I (Coll 1) and fibronectin (FN) fibers. Following IRI ${ }^{\text {(rep) }}$, the administration of FRCs restores the microarchitecture of the KLN and prevents renal fibrosis.

\section{Results}

The KLN is the site of $T$ cell activation and undergoes substantial structural changes driven by the activation of FRCs following IRI. The KLN was identified by injecting India ink into the abdominal aorta following clamp placement and was visualized using live light microscopy (Supplemental Figure 1A; supplemental material available online with this article; https://doi.org/10.1172/jci.insight.120546DS1). IRI was induced by a temporary clamp of the renal pedicle. To confirm the functional importance of the KLN to the inflammatory response following kidney IRI, Rag1 $1^{-/}$mice were injected with CellTrace Violet-labeled CD4 ${ }^{+} \mathrm{T}$ cells 2 days following unilateral IRI, and their KLNs were harvested. We observed marked increase in T cell proliferation in the KLN draining the ischemic kidney (KLN: IRI $\left.{ }^{(\mathrm{D} 2)}\right)$, as compared with either the KLN draining the nonischemic contralateral kidney

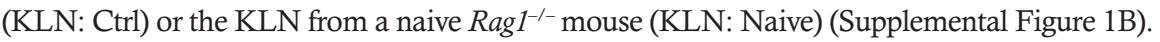

Next, we performed unilateral IRI using male C57BL/6 mice, and the kidneys and KLNs were harvested at 2 days and 30 days after IRI. We observed features of kidney injury 2 days following IRI in the ischemic kidney (Kidney: IRI $\left.{ }^{(\mathrm{D} 2)}\right)$, in contrast to the contralateral nonischemic kidney (Kidney: Ctrl) (Supplemental Figure 1C). By 30 days after IRI, the clamped kidney (Kidney: IRI $^{\left({ }^{D 30}\right)}$ ) had undergone repair, with no signs of tissue fibrosis (Supplemental Figure 1C).

Next, structural changes within the KLNs were assessed following IRI. H\&E staining of the KLN without IRI (KLN: Ctrl) showed typical cortical B cell zones (arrowhead) and paracortical T cell zones with clear medullary cords (asterisk). The KLN draining the ischemic kidney harvested 2 days after IRI (KLN: IRI ${ }^{(\mathrm{D} 2)}$ ) demonstrated a loss of compartmentalization of $\mathrm{T}$ and $\mathrm{B}$ cell zones, along with substantial cellular expansion, especially in the medulla (Figure 1A). Despite the resolution of the typical histologic changes associated with AKI in the renal parenchyma (Supplemental Figure 1C, Kidney: $\operatorname{IRI}^{(\mathrm{D} 30)}$ ), the KLN harvested at 30 days after IRI (KLN: IRI ${ }^{(\mathrm{D} 30)}$ ) demonstrated structural disorganization (Figure 1A). Interestingly, both IRI ${ }^{(\mathrm{D} 2)}$ and IRI ${ }^{(\mathrm{D} 30)} \mathrm{KLNs}$ displayed markedly augmented production of the antigen recognized by ER-TR7 antibody (ER-TR7) and $\mathrm{FN}^{+}$ECM by FRCs, in comparison with the Ctrl KLN (Figure 1A). The IRI ${ }^{(\mathrm{D} 2)}$ and IRI $\mathrm{I}^{(\mathrm{D} 30)}$ KLNs displayed an expanded, thickened, and nodular ECM, in contrast to the thin fibrillary pattern observed in the Ctrl KLN. The fluorescent signal of the ECM was increased in both the IRI ${ }^{(\mathrm{D} 2)}$ and $\mathrm{IRI}^{(\mathrm{D} 30)} \mathrm{KLNs}$, in comparison with the Ctrl KLN, as assessed by semiquantitative measurement (Supplemental Figure 1D).

$\mathrm{PDPN}^{+}$staining was used to study the architecture of FRCs located away from the lymphatic vasculature of the KLN (21). Immunofluorescence staining of FRCs in the IRI ${ }^{(\mathrm{D} 2)} \mathrm{KLN}$ demonstrated elongated cellular architecture. However, many FRCs lost their spindle-like shape and had an expanded cytoplasm in the IRI ${ }^{(\mathrm{D} 30)} \mathrm{KLN}$ (Figure 1B). Costaining of FRCs for PDPN and $\alpha$ smooth muscle actin $(\alpha \mathrm{SMA})$ suggested that FRCs underwent a transition to an activated phenotype in the $\mathrm{IRI}^{(\mathrm{D} 2)} \mathrm{KLN}$, an observation that was more prominent in the IRI ${ }^{(\mathrm{D} 30)} \mathrm{KLN}$ (Figure 1B). Mean fluorescence intensity of $\alpha \mathrm{SMA}$ in the IRI ${ }^{(\mathrm{D} 2)}$ and IRI ${ }^{(\mathrm{D} 30)}$ KLNs was significantly increased, as compared with the Ctrl KLN (Supplemental Figure 1D). Moreover, marked expansion and elongation of HEVs were observed in the $\mathrm{IRI}^{(\mathrm{D} 2)} \mathrm{KLN}$, and this pattern persisted in the IRI ${ }^{(\mathrm{D} 30)} \mathrm{KLN}$ (Figure 1B). Modest increases in the deposition of Coll I and the population of $\mathrm{F} 4 / 80^{+}$macrophages in the IRI ${ }^{(\mathrm{D} 2)} \mathrm{KLN}$ were more significant in the IRI $\mathrm{I}^{(\mathrm{D} 30)} \mathrm{KLN}$ (Supplemental Figure $1, \mathrm{D}$ and $\mathrm{E})$. The senescence marker $\mathrm{p} 16^{\mathrm{INK} 4 \mathrm{~A}}$ did not increase in either the IRI $\mathrm{I}^{(\mathrm{D} 2)}$ or IRI ${ }^{(\mathrm{D} 30)} \mathrm{KLNs}$, emblematic of the activated state of post-IRI FRCs (Supplemental Figure 1E) $(28,29)$. 
A

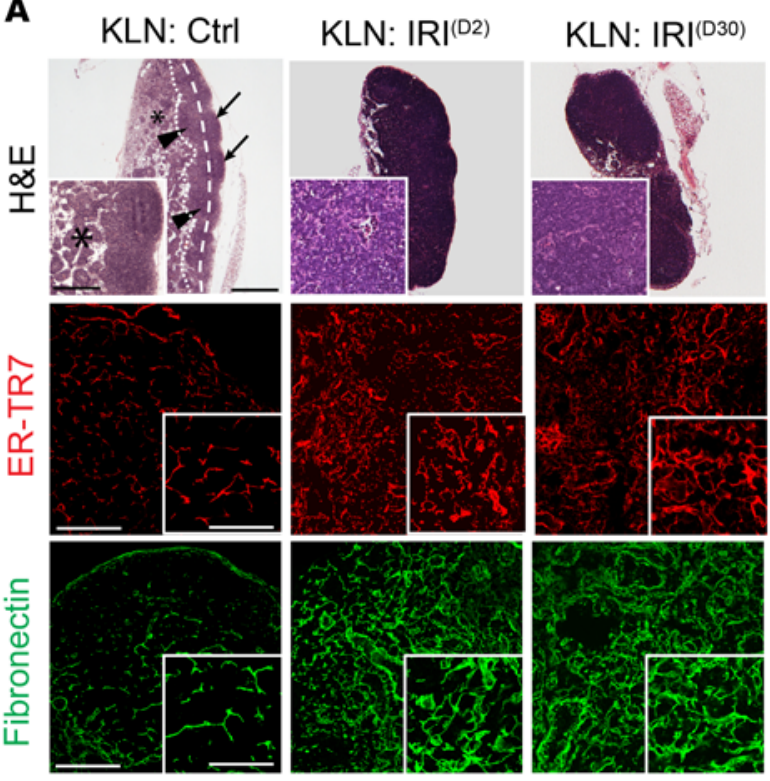

C

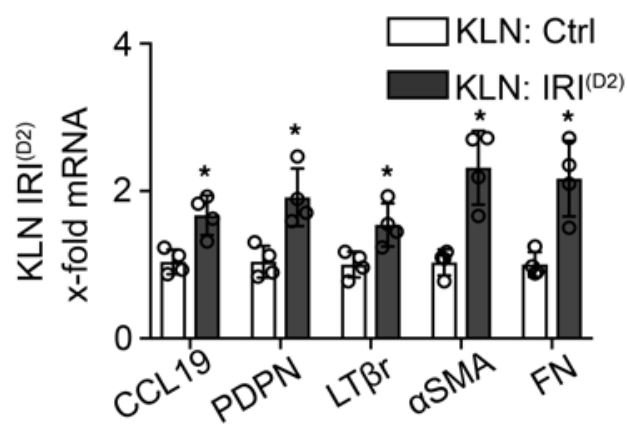

D
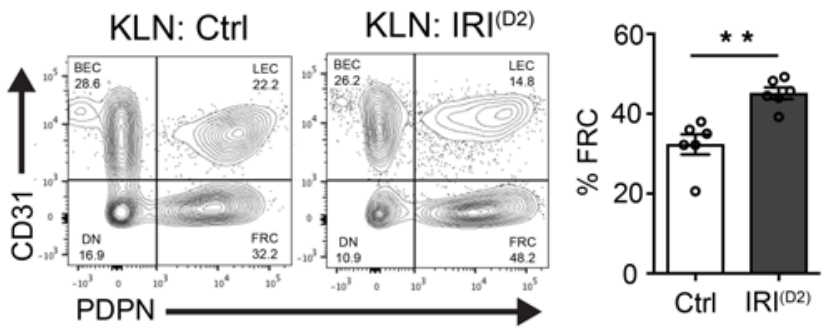

$\mathbf{F}$
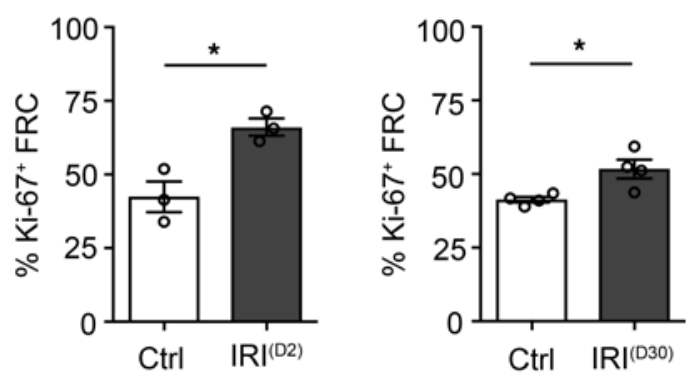
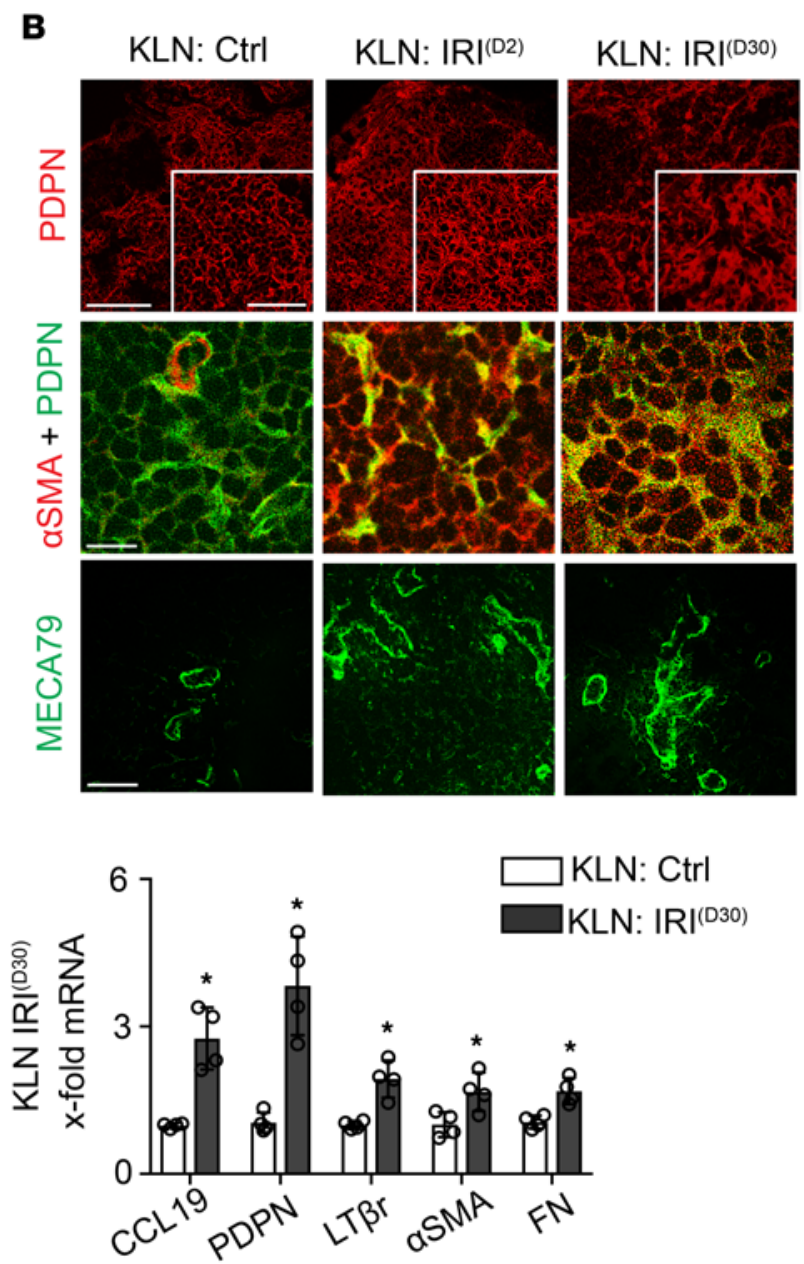

\section{E}
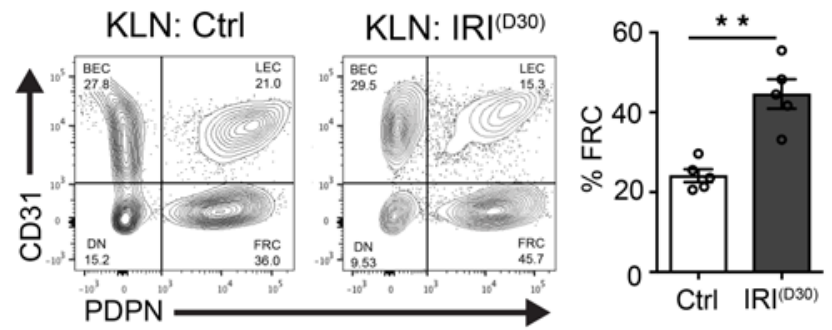
Figure 1. Activation of fibroblastic reticular cells (FRCs) results in major structural changes in kidney-draining lymph node (KLN) following ischemia-reperfusion injury (IRI) of the kidney. (A) KLN draining ischemic kidney shows loss of T and B cell zone differentiation, as compared with the KLN draining nonischemic kidney (KLN: Ctrl) at 2 days (KLN: IRI $\left.{ }^{(02)}\right)$ and 30 days (KLN: IRI $\left.\left.\right|^{(030)}\right)$ following IRI. Arrows point to cortical B cell zone, dashed line divides cortical from subcortical T cell zone (arrowheads), while dotted line divides subcortical zone from the medulla containing medullary chordae (inset: asterisk). At both time points, fluorescence reveals increased interstitial extracellular matrix (ECM) in KLN tissue: ER-TR7 and fibronectin (insets show thickened and nodular pattern). Scale bars: $500 \mu \mathrm{m}$ and $200 \mu \mathrm{m}$ (inset) for $\mathrm{H} \& \mathrm{E} ; 200 \mu \mathrm{m}$ and $100 \mu \mathrm{m}$ (inset) for ER-TR7 and fibronectin. (B) FRC signal (podoplanin, PDPN) is increased at 2 days (KLN: IRI $\left.\left.\right|^{(02)}\right)$ and 30 days (KLN: IRI $\left.\left.\right|^{(030)}\right)$ following IRI (inset shows enlarged cytoplasm). Costaining of PDPN and $\alpha$ smooth muscle actin ( $(S M A)$ suggests FRC transition in KLN following IRI of the kidney. High endothelial venules (HEVs) stained with MECA79, which labels peripheral node addressin on HEVs, show expansion and elongation 2 days and 30 days following IRI. Scale bars: $200 \mu \mathrm{m}$ and $100 \mu \mathrm{m}$ (inset) for PDPN; $100 \mu \mathrm{m}$ for $\alpha$ SMA+PDPN and MECA79. (C) Following IRI, KLN tissue expresses increased activated FRC gene transcripts, as assessed by qPCR ( $n=4 /$ group, mean \pm SEM). (D) Flow cytometry of KLNs shows increased CD45-PDPN+CD31- FRC percentage at 2 days following IRI (gated on CD45- cells, representative flow plots) ( $n=6 /$ group$^{-}$ mean \pm SEM). (E) Flow cytometry of KLNs shows increased CD45-PDPN+CD31-FRC percentage at 30 days following IRI (gated on CD45- cells, representative flow plots) ( $n=5$ /group, mean \pm SEM). (F) Flow cytometry of KLNs showed increased percentage of proliferating (Ki-67+) FRCs 2 days and 30 days following IRI in comparison with KLN of nonischemic kidney $\left(n=3-4\right.$ /group, mean \pm SEM). ${ }^{*} P<0.05$; ${ }^{* *} P<0.01$ by Student's $t$ test.

The expression of CCL19, PDPN, and lymphotoxin $\beta$ receptor (LT $\beta r)$ increased significantly in both the $\mathrm{IRI}^{(\mathrm{D} 2)}$ and IRI ${ }^{(\mathrm{D} 30)} \mathrm{KLNs}$, when normalized to the Ctrl KLN (Figure 1C). Concomitantly, the expression of profibrotic genes, including aSMA and FN, increased significantly (Figure 1C). Using flow cytometric analysis gated on $\mathrm{CD}^{-} 5^{-}$cells, we also confirmed a significant increase in the percentage of FRCs in both the $\mathrm{IRI}^{(\mathrm{D} 2)}$ and IRI ${ }^{(\mathrm{D} 30)} \mathrm{KLNs}$, as compared with the Ctrl KLN (Ctrl KLN vs. IRI ${ }^{(\mathrm{D} 2)} \mathrm{KLN}: 32.33 \% \pm 2.5 \%$ vs. $45.13 \% \pm$ $1.4 \%,{ }^{* *} P<0.01$; and $\mathrm{Ctrl}$ KLN vs. IRI ${ }^{(\mathrm{D} 30)} \mathrm{KLN}: 25.03 \% \pm 1.7 \%$ vs. $44.65 \% \pm 4.8 \%,{ }^{* *} P<0.01$; respectively) (Figure 1, D and E). Additionally, the percentage of lymphatic endothelial cells (LECs) was lower in both the IRI ${ }^{(\mathrm{D} 2)}$ and IRI ${ }^{\left({ }^{3} 30\right)} \mathrm{KLNs}$, in comparison with the $\mathrm{Ctrl} \mathrm{KLN}$, as assessed by flow cytometry (\%LEC: Ctrl KLN vs. IRI ${ }^{(\mathrm{D} 2)} \mathrm{KLN}, 20.43 \% \pm 1.5 \%$ vs. $13.78 \% \pm 1.8 \%,{ }^{*} P<0.05$; and Ctrl $\mathrm{KLN}$ vs. IRI ${ }^{(\mathrm{D} 30)} \mathrm{KLN}: 21.29 \%$ $\pm 1.6 \%$ vs. $15.36 \% \pm 1.6 \%,{ }^{*} P<0.05$; respectively), which is consistent with our findings from immunofluorescence (refer to Supplemental Figure 1E, Lyve1). The proliferation of FRCs was also significantly greater in both the IRI ${ }^{(\mathrm{D} 2)}$ and IRI ${ }^{(\mathrm{D} 30)} \mathrm{KLNs}\left(\% \mathrm{Ki}-67^{+}\right.$FRC: Ctrl KLN vs. IRI ${ }^{(\mathrm{D} 2)} \mathrm{KLN}, 42.40 \% \pm 5.2 \%$ vs. $66.10 \% \pm$ $2.9 \%,{ }^{*} P<0.05$; and $\mathrm{Ctrl} \mathrm{KLN}$ vs. IRI ${ }^{(\mathrm{D} 30)} \mathrm{KLN}: 41.27 \% \pm 0.9 \%$ vs. $51.66 \% \pm 3.2 \%,{ }^{*} P<0.05$; respectively) (Figure $1 \mathrm{~F}$ ). In conclusion, FRCs in the KLN were activated 2 days following IRI, and they remained activated 30 days following IRI, despite evidence of histological recovery from tubular injury in the kidney.

$I R I^{(r e p)}$ of the kidney causes senescence in KLN-resident FRCs. Since IRI is commonly recurrent, the impact of IRI ${ }^{\text {(rep) }}$ on progressive organ damage possesses high clinical relevance. We tested the hypothesis that IRI $^{\text {(rep) }}$ induces senescence in FRCs and leads to fibrosis in the KLN. To induce IRI ${ }^{\text {(rep) }}$, we performed IRI 3 times in C57BL/ 6 mice at 2-week intervals. Mice were then sacrificed 2 weeks following the third IRI (8 weeks after the first IRI). Histological analysis of the kidney following IRI ${ }^{(\text {rep })}$ demonstrated tubular injury and interstitial hypercellularity by H\&E, as well as interstitial fibrosis by Masson's trichrome staining (Supplemental Figure 2A). Analysis of gene expression in the kidney revealed an increase in proinflammatory markers, such as interferon $\gamma$ (IFN- $\gamma$ ), tumor necrosis factor $\alpha$ (TNF- $\alpha$ ), interleukin $1 \beta$ (IL-1 $\beta$ ), and IL-6, and fibrosis markers, such as FN, Coll I, and $\alpha$ SMA (Supplemental Figure 2B). The IRI ${ }^{\text {(rep) }}$ KLN showed marked morphologic changes, including loss of compartmentalization, loss of organization of $\mathrm{B}$ and $\mathrm{T}$ cell zones, and interstitial hypocellularity (Figure 2A). In addition, deposition of ER-TR7 and FN fibers increased, and their pattern of staining was patchy and lobular in the IRI ${ }^{\text {(rep) }} \mathrm{KLN}$ (Figure 2A, arrowheads), unlike the typical linear staining observed in Ctrl KLN (refer to Figure 1A, KLN: Ctrl). This finding correlated with an increase in FN gene expression in the IRI ${ }^{\text {(rep) }} \mathrm{KLN}$ (Supplemental Figure 2C).

Chronic injury leads to fibrosis, due to the formation of a fibrous scar resulting from activation of Coll I-producing myofibroblasts (30-33). We observed a marked increase in the staining of Coll I in the IRI ${ }^{\text {(rep) }} \mathrm{KLN}$ (Figure 2A, inset; dashed lines mark B cell zone). This finding was confirmed by gene expression analysis $\left({ }^{*} P<0.05\right.$, Supplemental Figure $2 \mathrm{C}$ ). Coll I can function as a macrophage chemoattractant (34). Indeed, we observed an increase in the infiltration of $\mathrm{F} 4 / 80^{+}$macrophages, in association with augmented deposition of Coll I fibers following IRI (rep) (Figure 2A). Macrophage-specific gene expression of F4/80 was also increased (Supplemental Figure 2C). PDPN-expressing FRCs appeared lobular with an edematous cytoplasm in the IRI (rep) $\mathrm{KLN}$ (Figure 2A), as compared with the Ctrl KLN (refer to Figure 1A, KLN: Ctrl). Moreover, flow cytometric analysis of the IRI ${ }^{\text {(rep) }}$ KLN revealed a significant decrease in the proportion of FRCs in the KLN, as expressed by the percentage of CD45- cells (Ctrl KLN vs. IRI (rep) $\mathrm{KLN}^{\text {: }}$ $35.80 \% \pm 3.4 \%$ vs. $11.34 \% \pm 1.8 \%,{ }^{* *} P<0.01$ ), and a marked decrease in the frequency of proliferating $\left(\mathrm{Ki}^{+} 7^{+}\right)$FRCs (Ctrl KLN vs. IRI Irep) KLN: $45.10 \% \pm 5.5 \%$ vs. $23.35 \% \pm 1.8 \%,{ }^{* *} P<0.01$ ) (Figure $2 \mathrm{~B}$ ). 

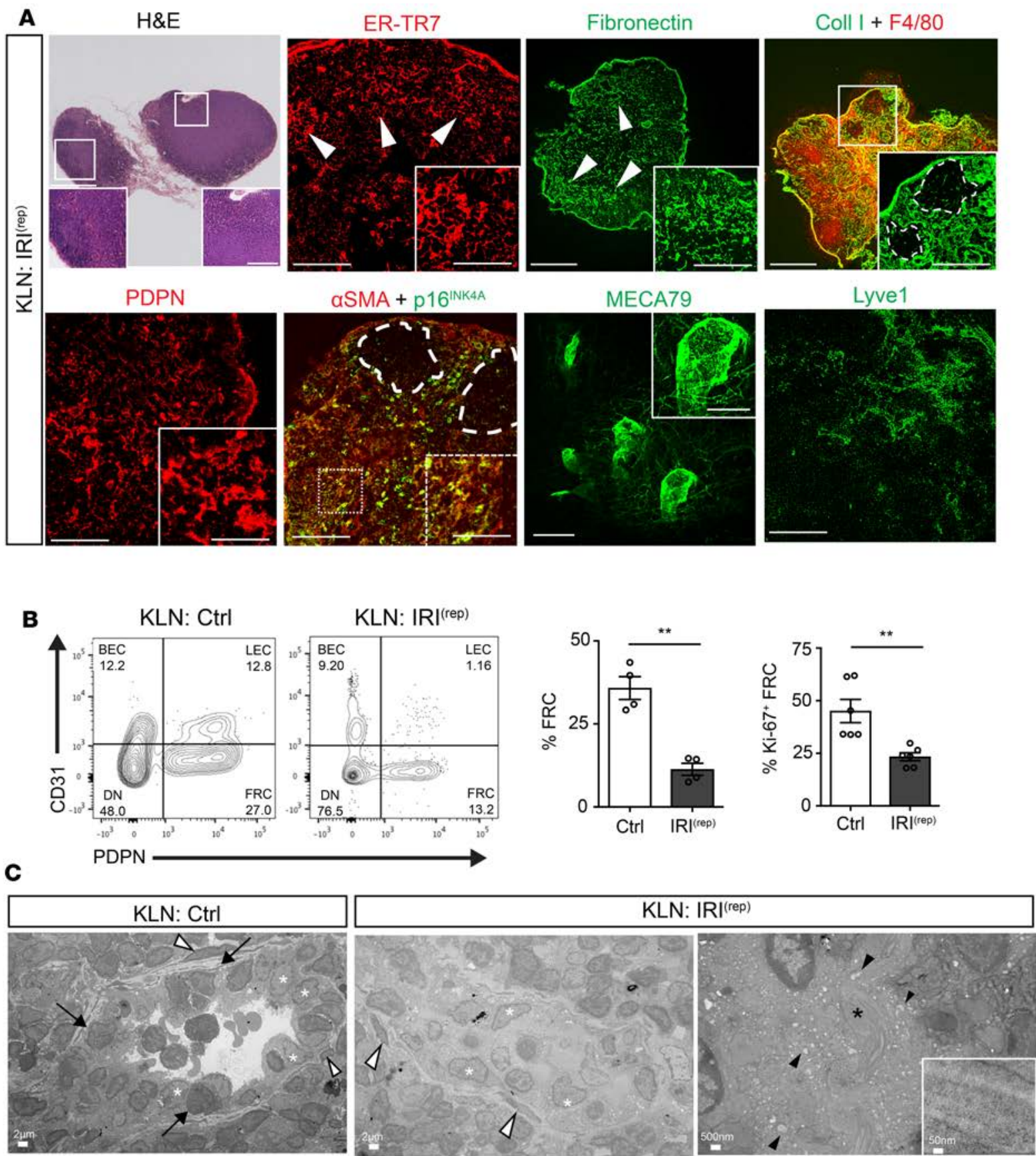

D

FRC: Ctrl
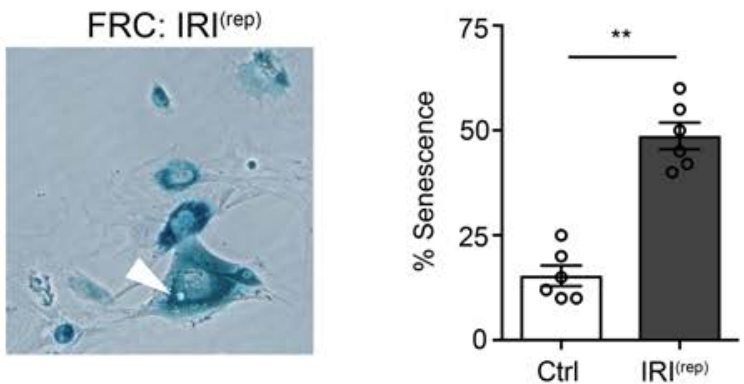
Figure 2. Repetitive renal IRI induces senescence in KLN-resident FRCs. KLNs were harvested 2 weeks after the induction of the third IRI (KLN: IRI (rep)). (A) Microscopy shows marked hypocellularity and loss of differentiation between cortical and subcortical zones. Both ER-TR7 and fibronectin staining insets illustrate areas of lobulated and thickened ECM. Arrowheads in ER-TR7 and fibronectin staining demonstrate areas of excessive ECM deposits with interstitial swelling. Collagen I matrix is increased, especially in subcortical areas, and ECM is expanded in the subcortical T cell zone, proximal to the B cell zone in costaining of KLN with Coll I and F4/80 (see dashed lines in inset). FRC staining demonstrates enlarged cytoplasms (inset in PDPN). Many KLN interstitial cells are stained with $\alpha$ SMA. Several of these cells are senescent (p16 $6^{\mathrm{INKA+}}$ ), especially in the subcortex (next to the dashed line); inset: close-up of senescent cells. HEV structure is markedly thickened (close-up image in inset). Lyve1 stain reveals regression of the lymphatic endothelial network. Scale bars: $500 \mu \mathrm{m}$ and $200 \mu \mathrm{m}$ (inset) for $\mathrm{H} \& \mathrm{E} ; 200 \mu \mathrm{m}$ and $100 \mu \mathrm{m}$ (inset) for ER-TR7, PDPN, and $\alpha \mathrm{SMA}+\mathrm{p} 16^{\text {INK } 4 \mathrm{~A} ;} 500 \mu \mathrm{m}$ and $100 \mu \mathrm{m}$ (inset) for fibronectin and CollI+F4/80; $100 \mu \mathrm{m}$ and $50 \mu \mathrm{m}$ (inset) for MECA79; $200 \mu \mathrm{m}$ for Lyve1. (B) Flow cytometric analysis of CD45- cells from KLNs following repetitive IRI (KLN: IRI (rep) $)$ or without repetitive IRI (KLN: Ctrl) shows significantly decreased percentages of CD45-PDPN+CD31- FRCs and proliferating (Ki67') FRCs, along with representative flow cytometry plots ( $n=4-6 /$ group, mean \pm SEM). (C) Electron microscopy of naive KLN shows HEV endothelium (white asterisks) and supporting perivascular FRCs (white arrowheads). Arrows indicate lymphocytes in active diapedesis into the interstitium of the KLN (KLN: Ctrl). KLN following repetitive IRI (KLN: $\mathrm{IRI}^{\text {(rep) })}$ shows obliteration of the HEV lumen with detachment of HEV cells (white asterisks) from the vessel's basement membrane and intrusion into the lumen. White arrowheads indicate perivascular FRCs. Some HEV cells show denser and smaller nuclei, along with shrunken cytoplasm, and HEV has intraluminal ECM deposits (black arrowheads). (D) FRCs from KLNs of mice with or without repetitive IRI were cultured in vitro and stained with $\beta$-galactosidase (senescence marker). Micrograph of FRCs from KLN following repetitive IRI showed senescent cells with lysosome-like vacuoles (white arrowhead) at a significantly higher percentage than FRCs from the KLNs of naive mice $\left(n=6\right.$ /group, mean \pm SEM). ${ }^{* *} P<0.01$ by Student's $t$ test.

Next, we sought to assess the cause of the decrease in frequency and proliferation of FRCs. Increased p16 ${ }^{\text {INK4A }}$ (Figure $2 \mathrm{~A}$, inset) staining was seen in areas where $\alpha$ SMA expression was more intense in the IRI ${ }^{\text {(rep) }}$ KLN (Figure 2A, dashed line; B cell zone areas). In contrast, senescence of FRCs was not observed at either day 2 or 30 after a single episode of IRI (refer to Supplemental Figure 1E, KLN: IRI ${ }^{(\mathrm{D} 2)}$ and KLN: IRI ${ }^{(\mathrm{D} 30)}$ ).

Furthermore, gene expression of $\alpha \mathrm{SMA}$, Coll I, FN, and F4/80 was increased in the IRI ${ }^{\text {(rep) }} \mathrm{KLN}$, as compared with the Ctrl KLN (Supplemental Figure 2C). The vessel walls of HEVs were markedly thickened in the IRI ${ }^{\text {(rep) }} \mathrm{KLN}$ (Figure 2A). In addition, Lyve ${ }^{+}$staining demonstrated a regression of LEC networks (Figure 2A), and flow cytometric analysis indicated that the proportion of LECs was decreased in the IRI ${ }^{\text {(rep) }}$ $\mathrm{KLN}$ in comparison with the $\mathrm{Ctrl} \mathrm{KLN}$, as represented by the percentage of CD45- cells (Ctrl $\mathrm{KLN}^{\text {vs. IRI }}{ }^{\text {(rep) }}$ KLN: $11.80 \% \pm 2.1 \%$ vs. $3.93 \% \pm 1.1 \%,{ }^{*} P<0.05$ ) (Figure $2 \mathrm{~B}$ ).

We then characterized the microstructure of the KLN using electron microscopy. A clear FRC-HEV unit, containing cuboidal HEV cells (Figure 2C, white asterisk) surrounded by spindle-like FRCs (Figure 2C, white arrowhead), was observed in the Ctrl KLN. Lymphocytes were observed trafficking between HEV cells into the interstitium of the KLN (Figure $2 \mathrm{C}$, black arrows). However, the IRI (rep) $\mathrm{KLN}$ demonstrated flattened perivascular FRCs and HEV cells, as well as detached HEVs obstructed by proteinaceous material (Figure 2C, KLN: $\mathrm{IRI}^{\text {(rep) }}$ ). The interstitial substructure revealed collagen fibrils (Figure $2 \mathrm{C}, \mathrm{KLN}$ : IRI ${ }^{\text {(rep) }}$, black asterisk, and inset) and numerous vacuoles (black arrowheads) in HEV cells, a finding suggestive of vascular injury (35).

To further assess senescence in the FRCs of KLNs following IRI ${ }^{\text {(rep) }}$, we isolated these cells, cultured them in vitro, and stained them for $\beta$-galactosidase, a commonly used in vitro senescence marker (Figure $2 \mathrm{D})$. The IRI ${ }^{(\text {rep })} \mathrm{KLN}$ contained a significantly higher ratio of $\beta$-galactosidase ${ }^{+} \mathrm{FRCs}_{\text {to }}$ total number of cells than the Ctrl KLN (Ctrl KLN vs. IRI ${ }^{\text {(rep) }} \mathrm{KLN}: 15.33 \% \pm 2.8 \%$ vs. $48.67 \% \pm 3.2 \%$, ${ }^{* *} P<0.01$ ) (Figure 2D) $(28,29)$. Senescent cells also form lysosome-like vacuoles $(36)$, and these were observed in abundance in the cytoplasm of cultured senescent FRCs (Figure 2D, arrowhead).

FRCs are activated via the lymphotoxin $\alpha$ signaling pathway. Next, we tested the hypothesis that $\mathrm{T}$ cells can activate FRCs through the action of membrane-bound lymphotoxin $\alpha$ (LT $\alpha$ ), as FRCs express LT $\beta r$ (37-39), the receptor to which LT $\alpha$ binds. We performed the adoptive transfer of T cells $\left(2.0 \times 10^{7}\right.$ cells $/$ mouse $)$ from either C57BL/6 (WT) or LT $\alpha$-KO mice (LT $\alpha^{-/}$) into $\mathrm{Rag}^{-/-}$mice 1 day prior to IRI. Two days after IRI, flow cytometric analysis indicated that the proportions of FRCs and proliferating (Ki-67+) FRCs were significantly higher in the KLN of the $\mathrm{Rag}^{-1-}$ mice that received an injection of WT T cells, in comparison with $\mathrm{LT} \alpha^{-/-} \mathrm{T}$ cells, as represented by percentages of CD $45^{-}$cells (\%FRC: WT vs. LT $\alpha^{-/-} \mathrm{KLN}, 17.00 \% \pm 1.7 \%$ vs. $7.22 \%$ $\pm 0.7 \%,{ }^{* *} P<0.01 ; \% \mathrm{Ki}^{*} 7^{+} \mathrm{FRC}$ : WT vs. $\mathrm{LT} \alpha^{-/-} \mathrm{KLN}, 32.95 \% \pm 3.4 \%$ vs. $15.66 \% \pm 1.4 \%$, ${ }^{* *} P<0.01$; respectively) (Figure 3A). Interestingly, the kidneys of $\mathrm{Rag}^{1^{-1-}}$ mice that received $\mathrm{LT} \alpha^{-/-} \mathrm{T}$ cells appeared to be relatively protected from IRI. H\&E staining of these kidneys revealed prominently fewer tubular casts and reduced thinning of the tubular epithelium (Figure 3B). In concordance with this, blood urea nitrogen (BUN) was significantly lower in $\mathrm{Rag}^{-1-}$ mice adoptively transferred with $\mathrm{LT} \alpha^{-/-} \mathrm{T}$ cells, as compared with the control group (WT vs. $\mathrm{LT}^{-/-}$: $95.57 \pm 6.242$ vs. $40.75 \pm 15.16 \mathrm{mg} / \mathrm{dl},{ }^{*} P<0.05$ ) (Figure $3 \mathrm{C}$ ).

Depletion of FRCs ameliorates AKI following IRI. FRCs recruit T cells to the LN via secretion of CCL19 $(11,23,40)$, where subsequently they become activated during IRI. Thus, we assessed whether the depletion 
of FRCs reduces kidney injury following IRI. To deplete FRCs, we used transgenic CCL19 $\mathrm{Cre} \times \mathrm{iDTR}$ mice, in which FRCs are depleted upon administration of diphtheria toxin (DT) $(15,41)$. To assess the potential impact of DT-induced depletion of FRCs in the kidney, we first measured the BUN of naive CCL19 ${ }^{\text {cre }} \times$ iDTR mice with or without DT administration. No significant difference in BUN between the groups was observed (data not shown). Furthermore, no change in the morphology of the glomeruli or tubules was noted following DT administration (Supplemental Figure 3, A and B). The expression of CCL19 in cultured podocytes was almost undetectable, as compared with the KLN (Supplemental Figure 3C). Then, DT was administrated to CCL19 ${ }^{\mathrm{Cre}} \times$ iDTR mice prior to bilateral IRI, and the mice were sacrificed 2 days following IRI. We observed a marked decrease in the signal of PDPN ${ }^{+}$FRCs in the KLN of CCL19 Cre $\times$iDTR mice, as compared with the KLN of WT mice (Figure 3D). FRC depletion prevented the expansion of the HEV network following IRI (Figure 3D). Quantification by flow cytometry showed a significant decline in the populations of both FRCs and HEV cells in the CCL19 ${ }^{\text {Cre }} \times$ iDTR KLN, in comparison with the WT KLN (Figure $3 \mathrm{E})$. The kidneys of CCL19 ${ }^{\mathrm{Cre}} \times \mathrm{iDTR}$ mice showed less severe acute tubular necrosis (ATN), as manifested by decreased tubular casts and reduced tubular thinning (Figure 3F). Depletion of FRCs also led to a marked decrease in macrophage infiltration into the kidneys following IRI (Figure 3F, lower panel). Moreover, the cortices in the kidneys of FRC-depleted mice were thickened significantly in comparison with the WT kidneys 2 days following IRI, as assessed by a cortical thickness score (WT vs. CCL19 ${ }^{\text {Cre }} \times$ iDTR: $1.6 \pm 0.48$ vs. $2.75 \pm 0.21,{ }^{*} P<0.05$ ) (Figure $3 \mathrm{G}$, left). Finally, the BUN of CCL19 ${ }^{\text {Cre }} \times$ iDTR mice was commensurately lower than the WT mice $\left(68.03 \pm 4.1\right.$ vs. $29.0 \pm 4.5 \mathrm{mg} / \mathrm{dl}$, $\left.{ }^{* *} P<0.01\right)$ (Figure $3 \mathrm{G}$, right).

Next, we investigated whether FRC depletion altered the proinflammatory $\mathrm{T}$ cell profile in the KLN following IRI. At day 2 following IRI, the absolute numbers of proinflammatory CD $4^{+} \mathrm{IFN}-\gamma^{+} \mathrm{T}$ cells (WT vs. CCL19 ${ }^{\text {Cre }} \times$ iDTR: $3,112.4 \pm 1,110$ vs. $\left.892.1 \pm 191.8,{ }^{*} P<0.05\right)$ and activated CD4 ${ }^{+} C D 44^{\text {hi }}$ T cells (WT vs. CCL19 ${ }^{\text {Cre }} \times$ iDTR: 7,602.1 $\pm 1,405$ vs. $\left.1,895.2 \pm 648.2,{ }^{*} P<0.05\right)$ were significantly lower in the KLN of the CCL19Cre $\times$ iDTR mice in comparison with the WT mice (Figure 3H). These studies suggest a vital role of KLN-resident FRCs in the pathogenesis of the acute inflammatory response in the kidney following IRI.

$L T \beta r$-Ig treatment enhances renal fibrosis following IRI. FRCs can stimulate the immune response through the recruitment of $\mathrm{T}$ cells, but they can also inhibit the immune response, through their ability to produce immunoregulatory molecules (23). Proper healing of organs following an initial ischemic insult relies on the activation of regulatory immune responses to counteract the proinflammatory processes $(9,42)$. We tested the hypothesis that chronic suppression of the LT $\alpha$ signaling pathway interferes with the healing phase following renal IRI. Prolonged treatment with DT over several weeks is associated with significant toxicity. Therefore, we treated mice that underwent renal IRI with LT $\beta r$-immunoglobulin fusion protein (LT $\beta r-I g)$. LT $\beta r$ plays an important role in the function and maturation of FRCs (43). LT $\beta r$-Ig functions as a decoy protein, interfering with the interaction between LT $\alpha$ and LT $\beta r$ (44). Starting 4 days prior to bilateral renal IRI, LT $\beta r$-Ig was injected intraperitoneally (i.p.) every 2 days, including the day of surgery. After surgery, it was given 3 times weekly, until the mice were sacrificed 30 days following IRI. Experimental control mice were treated with control IgG. Upon histological assessment at 30 days after IRI, we observed that the kidneys from the mice treated with LT $\beta r$-Ig did not display signs of recovery from IRI, in comparison with the control group (Figure 4A). LT $\beta r$-Ig-treated mice had increased tubulointerstitial fibrosis (Figure 4A), with a marked increase in myofibroblast transition, as evidenced by $\alpha \mathrm{SMA}$ expression in the interstitium (Figure 4A). Kidney tissue showed excessive layering of FN and increased infiltration with $\mathrm{F} 4 / 80^{+}$macrophages, following treatment with LT $\beta r-I g$ (Figure 4A). Similarly, KLNs of mice injected with LT $\beta$ r-Ig displayed signs of fibrosis, including a marked increase in FN expression (Figure 4B). In keeping with the histological appearance, the BUN was significantly higher on day 30 in the mice that received LT $\beta r-I g$, as opposed to the control IgG (IgG vs. LT $\beta r-I g: 14.53 \pm 3.4$ vs. $26.63 \pm$ $5.2 \mathrm{mg} / \mathrm{dl},{ }^{*} P<0.05$ ) (Figure $4 \mathrm{C}$ ). These experiments suggest that the inhibition of FRCs in the KLN with LT $\beta r-I g$ hinders renal repair following IRI and leads to fibrosis.

FRC infusion alleviates renal fibrosis following recurrent IRI. Next, we hypothesized that the infusion of exogenous FRCs could restore the anatomy of the KLN and prevent renal scarring following IRI ${ }^{\text {(rep) }}$.

FRCs were isolated from the LN and expanded in vitro as previously described (45). To confirm the homing of FRCs to the KLN following IRI, we labeled FRCs with IR800-loaded PLGA nanoparticles (FRCIR800-NPs). Following injection, FRC-IR800-NPs localized preferentially to the KLN draining the ischemic kidney (Figure 5A, white arrow), as compared with the KLN draining the nonischemic kidney (Figure 5A, blue arrow); no signal was detected in the nondraining popliteal LN (Figure 5A, white arrowheads). 
A
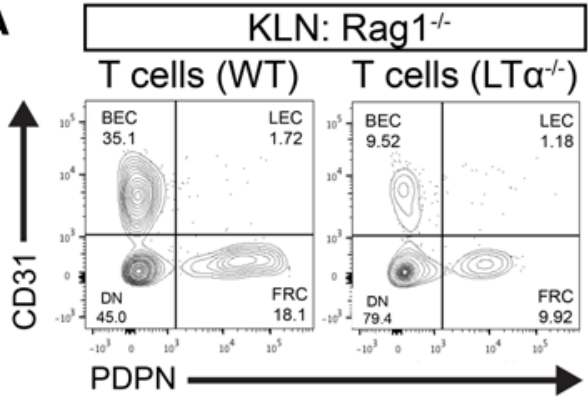

B

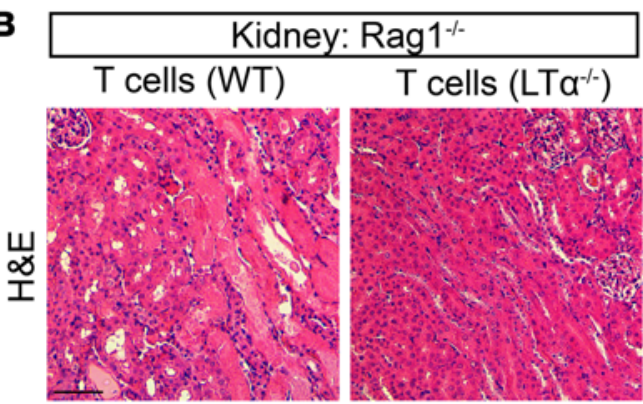

D

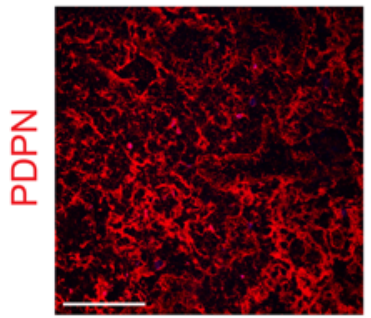

KLN: CCL19 ${ }^{\text {Cre }} \times$ iDTR

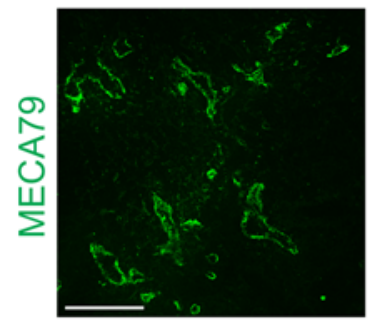

$\mathbf{F}$

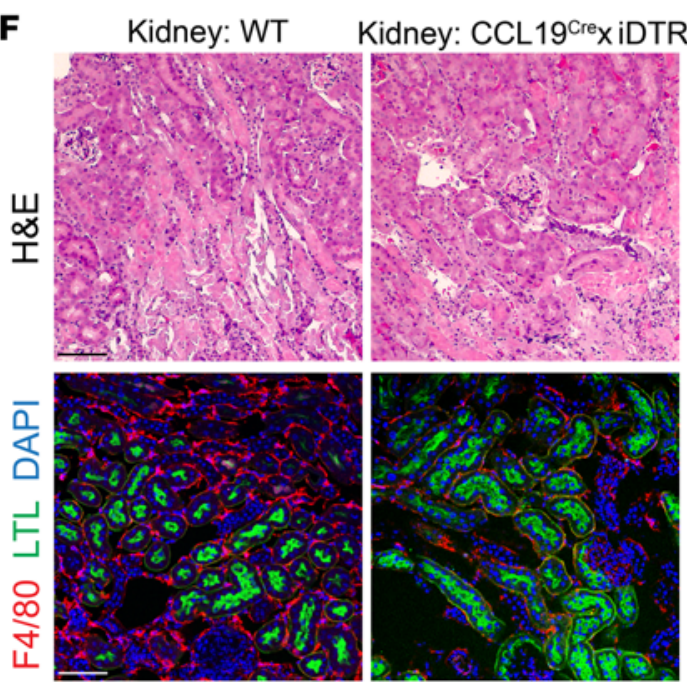

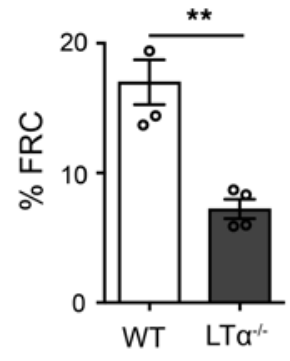

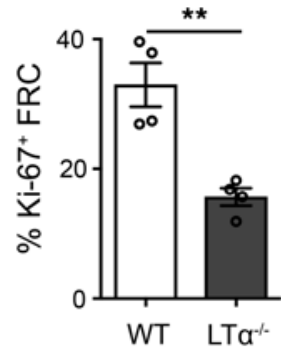

C

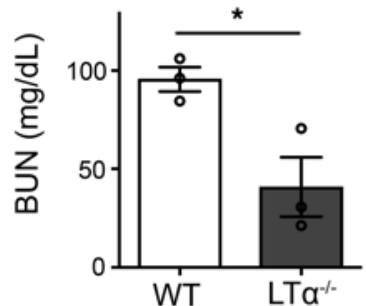

$\mathbf{E}$
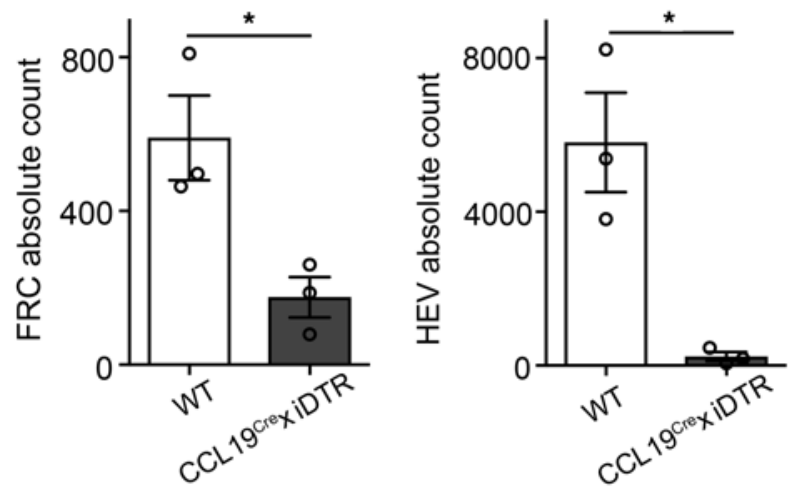

G
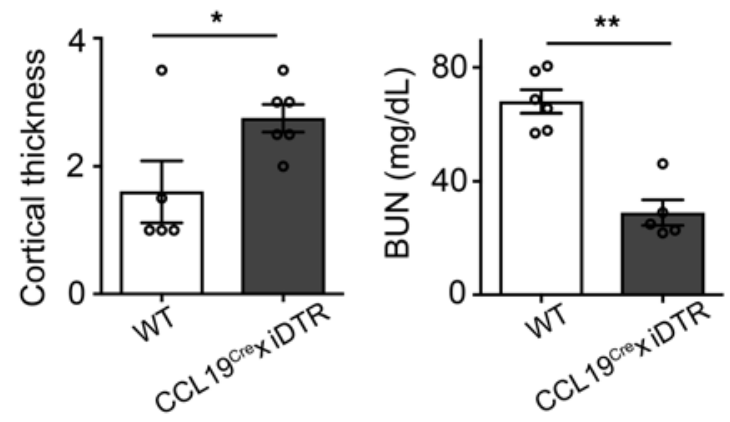

$\mathbf{H}$

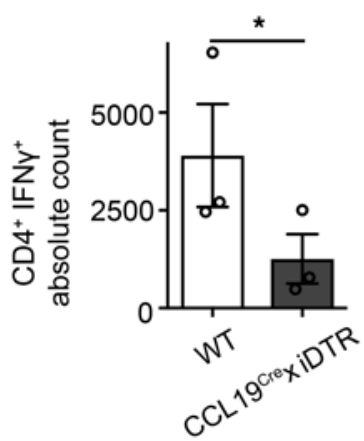


Figure 3. FRCs play a crucial role in the augmentation of immune activation following IRI. (A) Flow cytometric analysis shows a higher percentage of CD45-PDPN+CD31- FRCs and proliferating (Ki67+) FRCs in KLNs harvested 2 days following IRI from Rag1/- mice injected with WT T cells than KLNs from those injected with LT $\alpha^{-1-}$ T cells, along with representative flow cytometry plots (gated on CD45- cells) ( $n=3-4 /$ group, mean \pm SEM). (B) H\&E staining (scale bar: 75 $\mu \mathrm{m}$ ) of kidney tissue from $\mathrm{Rag}^{-1 /-}$ mice following IRI demonstrates renal protection upon adoptive T cell transfer from $\mathrm{LT} \alpha^{-/-}$mice, along with (C) lower BUN, as compared with Rag1/- mice that received T cells from WT mice ( $n=3 /$ group, mean \pm SEM). (D) Immunofluorescence of KLN shows marked decrease in $\mathrm{PDPN}^{+}$FRCs in DT-treated CCL19 ${ }^{\text {re }} \times$ iDTR mice as compared with untreated WT mice. Paucity in HEV abundance and abrogation of architectural elongation is also shown. Scale bars: $200 \mu \mathrm{m}$ for PDPN, $100 \mu \mathrm{m}$ for MECA79. (E) Absolute cell counts of FRCs and HEVs are significantly lower in the KLNs of CCL19cre $\times$ iDTR mice, in comparison with the KLNs from WT mice, as determined by flow cytometric analysis ( $n=3$ /group, mean \pm SEM). (F) FRC depletion leads to amelioration in kidney damage after IRI. H\&E staining shows less tubular injury in CCL19Cre $\times$ iDTR mice, as compared with WT mice. F4/80 staining indicates tissue inflammation is markedly decreased in CCL19 ${ }^{\text {cre }} \times$ iDTR mice after IRI. LTL identifies proximal tubules. Scale bars: $75 \mu \mathrm{m}$. (C) Left panel: Mean cortical thickness of kidneys in FRC-depleted mice was higher than in WT mice following IRI ( $n=6 /$ group, mean \pm SEM). Right panel: CCL19 ${ }^{\text {Cre }} \times$ iDTR mice are protected from IRI, as indicated by lower BUN (mg/dl) ( $n=6 /$ group, mean \pm SEM). (H) Left panel: Absolute count of CD4 ${ }^{+}$IFN- $\gamma^{+}$T cells in KLN is significantly lower in CCL19 1 cre $\times$ iDTR mice in comparison with WT mice following IRI. Right panel: Absolute count of CD4 ${ }^{+C D 44^{\text {hi }}}$ T cells in KLN is significantly lower in CCL19 ${ }^{\text {cre }} \times$ iDTR mice in comparison with WT mice $\left(n=3-4 /\right.$ group, mean \pm SEM). ${ }^{*} P<0.05$; ${ }^{*} P<0.01$ by Student's $t$ test.

Next, we subjected mice to bilateral renal IRI ${ }^{\text {(rep) }}$ as above -3 occasions of IRI, separated by intervals of 2 weeks - and injected them with FRCs (passage 3 to 4) intravenously, immediately prior to each renal insult. Flow cytometric analysis of KLNs confirmed homing of FRCs to the KLNs after IRI (vehicle vs. FRC: $3,640 \pm 933.2$ vs. $8,985 \pm 1,432,{ }^{* *} P<0.01$ ) (Figure $5 \mathrm{~B}$ ). Two weeks following the final insult, the mice that received exogenous FRCs displayed decreased kidney injury on H\&E staining, as manifested by reductions in tubular dilation (Figure 5C, arrowheads), interstitial expansion, cellularity (Figure 5C, arrows), and tissue fibrosis by Masson's stain (Figure 5C), as compared with the vehicle-treated mice. Immunofluorescence of kidneys from FRC-treated mice also displayed lower FN density and diminished macrophage infiltration, as compared with the control group (Figure 5D). Semiquantitative analysis of the fluorescence signals confirmed these findings in FRC-treated mice (vehicle vs. FRC: FN, $35.68 \pm 4.4$ vs. $19.02 \pm 2.8,{ }^{* *} P<0.01$; macrophage, $24.92 \pm 4.3$ vs. $8.63 \pm 3.1,{ }^{*} P<0.05$ ) (Figure $5 \mathrm{E}$ ). Flow cytometric analysis of infiltrating cells in the kidney revealed a marked reduction in the macrophage population in the kidneys of mice treated with FRCs (vehicle vs. FRC: $14.96 \times 10^{4} \pm 1.9 \times 10^{4}$ vs. $8.04 \times 10^{4} \pm 1.5 \times 10^{4}$, ${ }^{* *} P$ $<0.01$ ) (Figure 5F). Interestingly, histological assessment of the KLNs from the FRC-treated mice showed improved architecture with well-demarcated B cell, T cell, and medullary zoning, as compared with the vehicle-treated mice (Figure 5G).

\section{Discussion}

FRCs play an important role in supporting the structure of the $\mathrm{LN}$, and their functions include the recruitment of immune cells, production of ECM, and maintenance of HEV integrity (23, 46-48). Furthermore, FRCs participate in LN recovery and remodeling following acute infections (23). These functions are critically important to the generation of an antigen-specific immune response (11, 12, 21, 23). Although FRCs are vital in the control of antiviral immunity $(23,41,49)$, their role in the pathogenesis of immune responses following ischemic organ injury is unknown. We have found that the KLN is the site of $\mathrm{T}$ cell activation following IRI of the kidney, as we observed a considerable expansion of immune cells. Also following IRI, FRCs in the KLN proliferated and increased the production of ECM. Additionally, we noted an expansion of the HEV cell population. These histological changes in the KLN persisted despite full renal recovery following a solitary episode of IRI. This lack of full recovery could be due to ongoing inflammatory processes.

Repetitive ischemic kidney injury is known to contribute to the progression of chronic kidney disease (50). Moreover, persistent stimulation of FRCs has been demonstrated to induce phenotypic changes, whereby FRCs produce large amounts of collagen, leading to fibrosis in the $\mathrm{LN}(49,51)$. Indeed, following IRI ${ }^{\text {(rep) }}$, we found that the KLN demonstrated excessive deposition of ECM fibers containing FN and Coll I, as well as decreased density of FRCs. While the pattern of ER-TR7 and FN in the nonischemic KLN is continuous and linear, a lobular pattern of FN deposition is observed in the KLN following IRI. We also noted a significant increase in macrophage frequency, in keeping with prior observations by others $(52,53)$. FRCs also maintain the integrity of HEVs (54), and we observed marked obliteration of HEVs following IRI, with proteinaceous and fibrous material laid outside these vessels. The HEV is central to determining the fate of immune responses through its important role in facilitating the entrance of immune cells into the LN.

Collagen and other ECM fibers, such as FN, accumulate in fibrotic tissues (55). The accumulation of collagen and $\mathrm{FN}$ in the post-IRI ${ }^{\text {(rep) }} \mathrm{KLN}$ shares pathological characteristics with fibrosis within other organs (56). Excessive deposition of collagen results in defects in the mechanical properties of organs, 
A
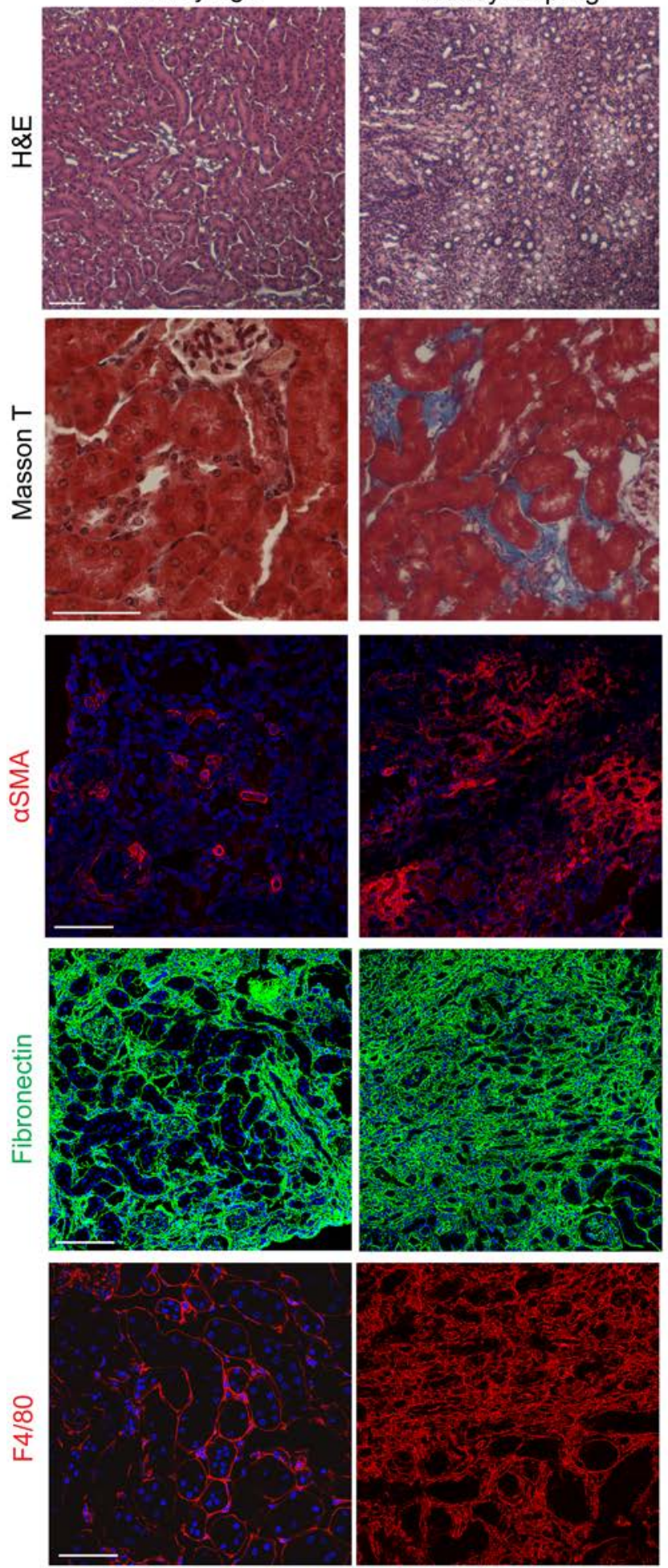

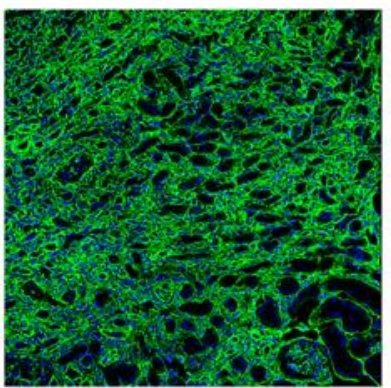

Kidney: LTßr-lg
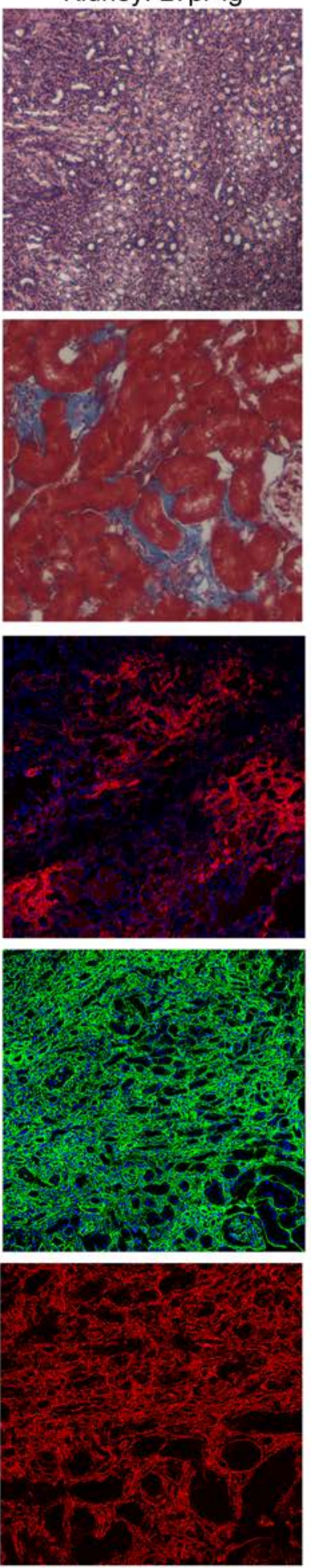

B
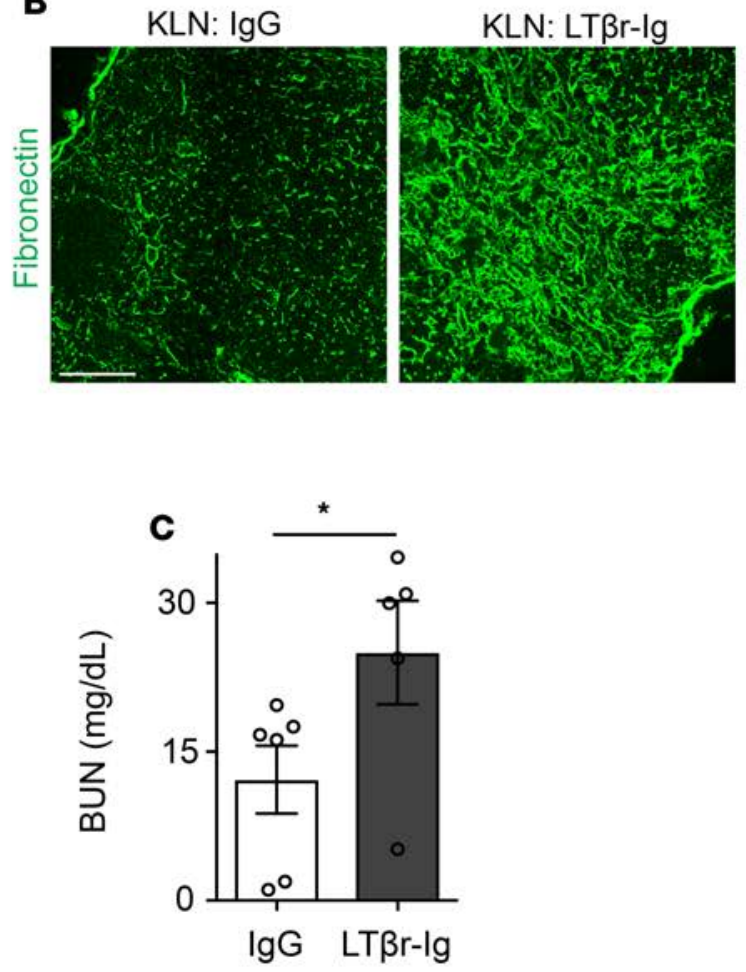

Figure 4. LT $\beta$ receptor-immunoglobulin (LT $\beta r$-Ig) treatment enhances renal fibrosis following IRI. (A) Ischemic kidney from mouse treated with $L T \beta r$-lg fails to recover histologically (H\&E) 30 days following IRI and shows tissue fibrosis (Masson $T$ ), in comparison with the kidney from mouse treated with control IgG. Thirty days following IRI, the ischemic kidney from mouse treated with LT $\beta r$-lg shows increased staining of $\alpha$ SMA, fibronectin, and $\mathrm{F} 4 / 80$, as compared with the kidney from mouse treated with control IgG. Scale bars: $75 \mu \mathrm{m}$. (B) Thirty days following IRI, KLN from mouse treated with LT $\beta r$-lg shows marked increase in fibronectin staining, as compared with KLN from mouse treated with control IgG. Scale bar: $200 \mu \mathrm{m}$. (C) Thirty days following IRI, BUN is significantly higher in mouse receiving LT $\beta r-I g$, as compared with mouse treated with control IgG ( $n=5-6 /$ group, mean $\pm \mathrm{SEM}) .{ }^{*} P<0.05$ by Student's $t$ test.

such as increased stiffness (57). Given the importance of contractility of FRCs to the expansion of the $\mathrm{LN}$ in a mounting immune response, it would be of considerable interest to assess the effect of altered stiffness of the KLN following IRI ${ }^{\text {(rep) }}$ on renal outcomes (58).

FRCs share many features with mesenchymal stem cells (MSCs) (i.e, CD73 and CD90 expression). Though cellular senescence was originally described in fibroblasts, MSCs under stress have similarly been demonstrated to undergo senescence $(59,60)$. Senescent cells experience growth arrest, but they maintain 
A
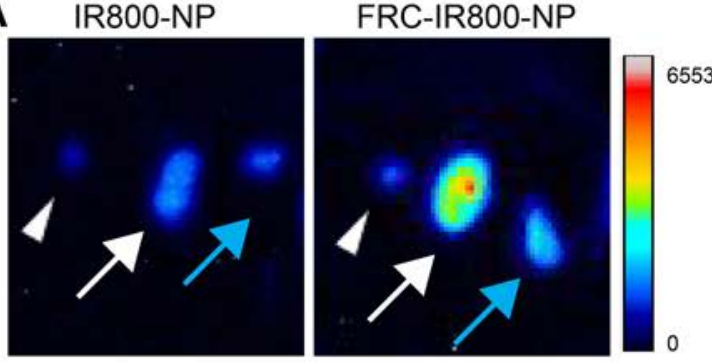

C
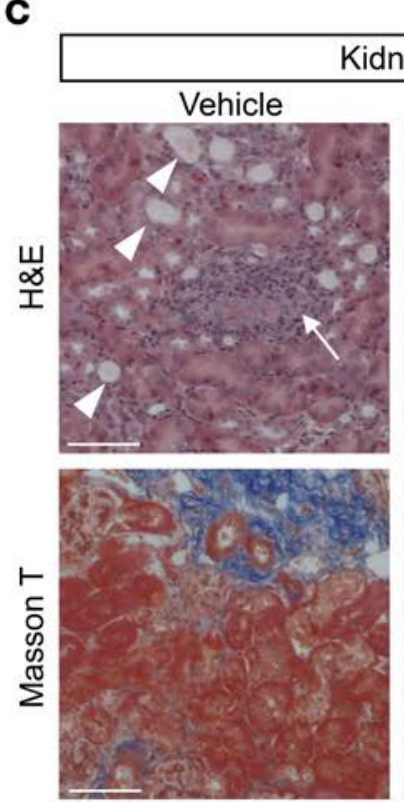

E

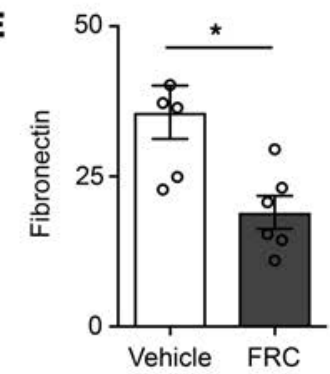

$\mathbf{F}$
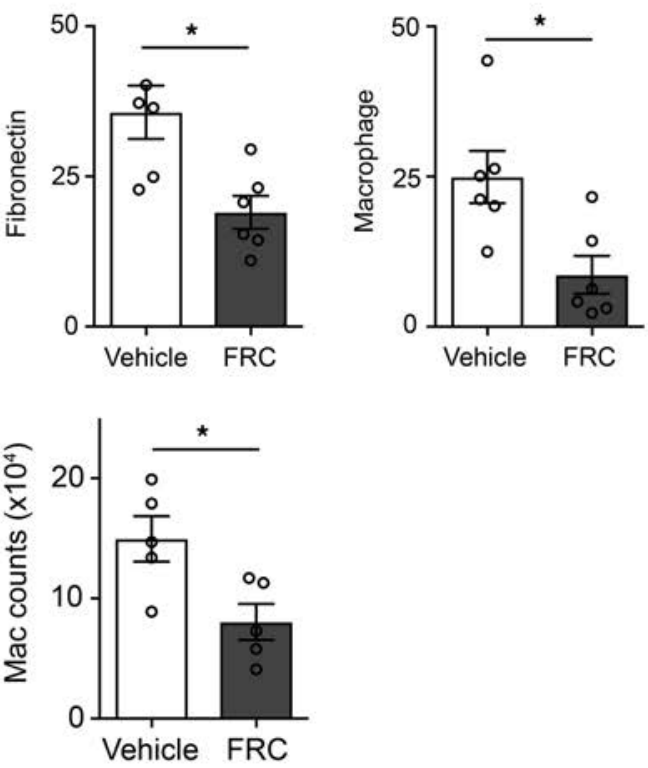

B

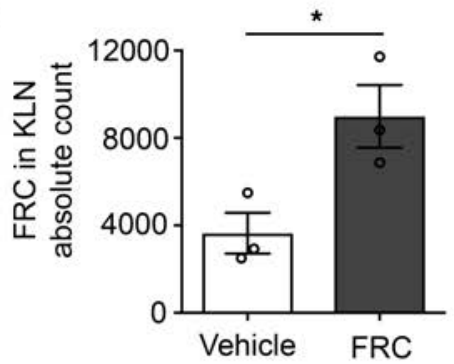

D

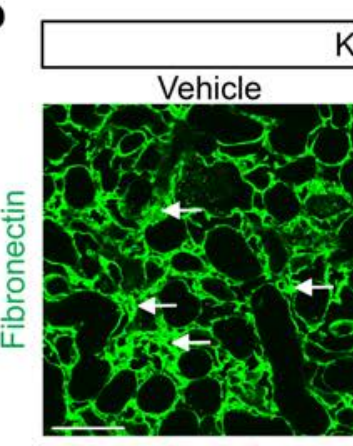

Kidney
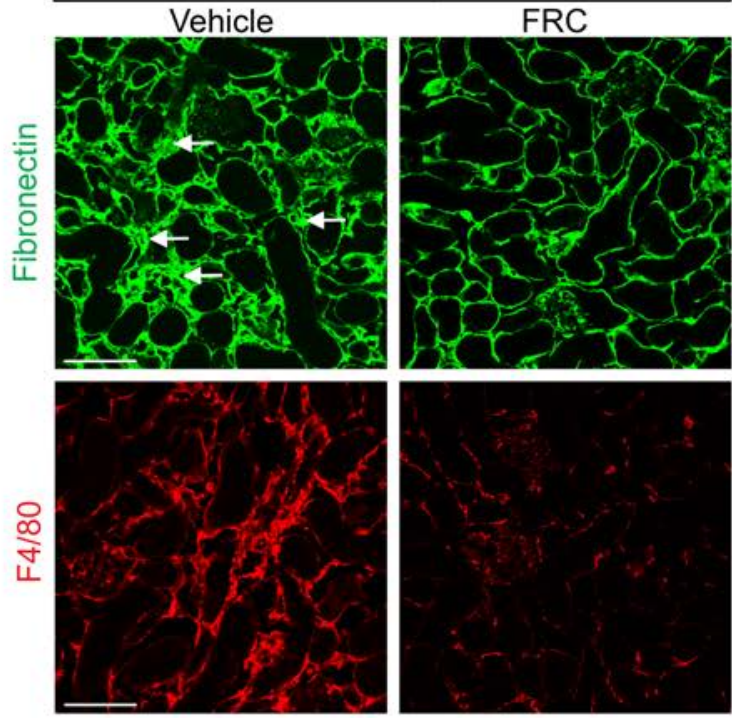

G

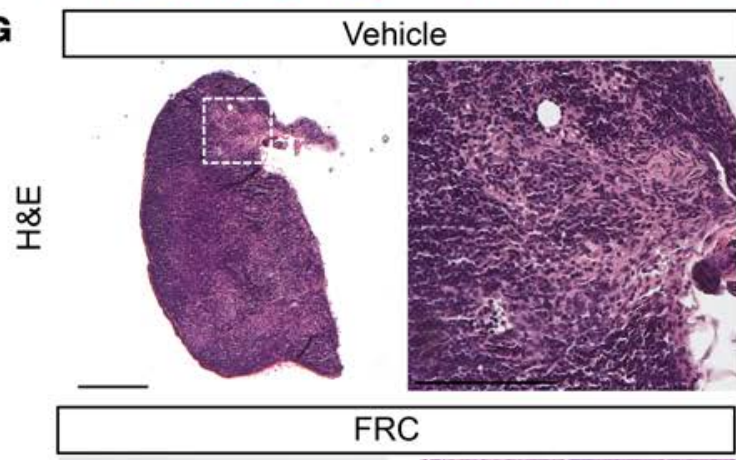

$\underset{\Upsilon}{\varpi}$
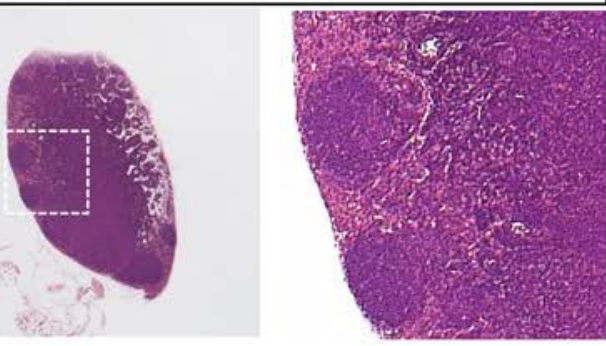
Figure 5. Injection of FRCs ameliorates chronic renal injury following repetitive IRI. (A) IR800-NP-labeled FRCs preferentially traffic to KLN draining injured kidney (arrowhead: popliteal LN; white arrow: KLN draining ischemic kidney; blue arrow: KLN draining non-ischemic kidney). (B) Flow cytometric analysis demonstrates that injection of FRCs at time of recurrent IRI leads to increased FRC population in KLN in comparison with injection of vehicle ( $n$ = 3/group, mean \pm SEM). (C) Light microscopy shows amelioration of kidney injury in mice injected with FRCs at the time of recurrent IRI, as compared with mice injected with vehicle (arrowheads: cast-filled and dilated tubules; arrow: interstitial expansion and hypocellularity). Masson's trichrome staining shows decreased fibrosis in FRC-treated mice. Scale bars: $75 \mu \mathrm{m}$. (D) Substantial declines in fibronectin density and F4/80+ macrophage infiltration of kidney is observed following FRC injection at the time of recurrent IRI. Scale bars: $75 \mu \mathrm{m}$. (E) Semiquantitative analysis of the corresponding fluorescent signals in kidney tissue shows significantly lower signals in the FRC-treated group ( $n=5$-6/group, mean \pm SEM). (F) Absolute count of kidney-infiltrating macrophages following IRI, as measured by flow cytometry, confirms a significant drop in macrophage recruitment with FRC treatment ( $n=5 /$ group, mean \pm SEM). (C) FRC injection prevents architectural changes in KLN following IRI. Scale bars: $500 \mu \mathrm{m}$ (left) and $200 \mathrm{~mm}$ (right). ${ }^{*} P<0.05$ by Student's $t$ test.

metabolic activity and can produce a variety of molecules, including cytokines. This cellular state is referred to as the senescence-associated secretory phenotype and could potentially stimulate a proinflammatory milieu within the KLN (61). FRCs isolated from the KLNs of mice experiencing repetitive ischemia displayed a significant decline in proliferation and experienced increasing growth arrest in cultures. They also appeared enlarged and crowded with vacuoles, and they exhibited senescence-associated $\beta$-galactosidase activity (62).

FRCs express LT $\beta$ r, which allows them to interact with LT $\alpha$. This interaction has been found to be crucial to the development of LNs (43). It should be noted that LT $\alpha$ on the surface of immune cells exists as a heterotrimer of LT $\alpha 1 / \beta 2$. LT $\alpha$ is also present in a secreted form as homotrimeric LT $\alpha 3$, which acts on TNFR1/2, not LT $\beta r$ (63). Here, we tested the hypothesis that the activation of FRCs occurs via signaling through the LT $\alpha / \mathrm{LT} \beta \mathrm{r}$ pathway. Adoptive transfer of $\mathrm{T}$ cells from $\mathrm{LT} \alpha^{-/-}$mice into $R a g 1^{-/-}$mice protected against kidney injury following IRI, as compared with $R a g 1^{1^{--}}$mice that received T cells from WT mice, indicating the crucial role of LT $\alpha$ in the activation of FRCs by T cells following IRI.

Next, we tested the hypothesis that the depletion of FRCs reduces AKI following IRI through the utilization of a transgenic CCL19 ${ }^{\text {Cre }} \times$ iDTR mouse model, as previously described $(15,64)$. CCL19-Cre $\times$ Rosa26-EYFP reporter mice were generated by crossing the CCL19-Cre line with Rosa26-enhanced yellow fluorescent protein (EYFP) mice, which allowed for the identification of FRCs as well as their depletion, through the addition of the DTR gene (15). We found that animals depleted of FRCs showed less severe renal injury, along with fewer kidney-infiltrating inflammatory cells, in the acute phase following IRI. The KLN of FRC-depleted mice also contained significantly fewer activated proinflammatory $\mathrm{T}$ cells. This difference could be due to the importance of CCL19-bearing FRCs to the recruitment of immune cells via HEVs to the KLN in the setting of IRI $(11,12,23)$.

CCL19 has been reported to be expressed by cultured podocytes and kidneys suffering from chronic glomerular diseases $(65,66)$. Given the acuity of our model, it is unlikely the effect we noted on renal function following DT therapy resulted from depletion of CCL19-expressing cells of normal kidneys. Furthermore, the glomeruli and tubules of the kidney did not demonstrate any significant histological changes following DT therapy in CCL19 $\mathrm{cre} \times \mathrm{iDTR}$ mice.

In order to tightly control the microenvironment of LN, FRCs carry the potential to have both proinflammatory and regulatory functions $(23,67)$. The initiation of immunoregulatory processes mediated by FRCs within the KLN likely promotes organ healing by suppressing inflammatory responses (68-71). Because chronic depletion of FRCs with DT is challenging due to the high toxicity rate of long-term use, we sought to block the LT $\beta r$ pathway to assess whether this alteration interferes with the healing phase of renal IRI.

LT $\beta$ r-Ig was tested for its therapeutic efficacy in an immune complex glomerulonephritis model (72). LT $\beta$ r-Ig was efficacious in reducing proteinuria, when it was administered from 3 to 7 weeks following initiation of this disease model. However, earlier LT $\beta$ r-Ig treatment resulted in high mortality. In addition, beginning the treatment at 5 weeks resulted in no significant effect on renal outcomes. In keeping with this observation, Bromberg's group has shown that LT $\beta$ r-Ig treatment significantly altered stromal subsets of LNs, eroding transplant tolerance and resulting in organ fibrosis (73). Similarly, in our model, LTßr-Ig treatment augmented renal fibrosis following IRI. These data could suggest dynamism in the role that signaling through the LT $\beta r$ pathway plays at different stages of immune-mediated diseases (43).

It is important to note while interpreting these findings that LT $\beta \mathrm{r}$ and its ligands can bind to several other types of receptors and ligands, which adds to the complexity of the system. For instance, LIGHT is a tumor necrosis factor-related cytokine that also binds to LT $\beta$ r. Interestingly, LT $\beta r$-Ig binds to both heterotrimeric membrane-bound LT $1 \beta 2$ and LIGHT (74). Future studies are needed to further delineate the relative role of LIGHT (and its receptors HVEM and decoy receptor 3 ) in the patho- 
genesis of fibrosis in the LN following IRI ${ }^{\text {(rep) }}$. Future studies are also needed to uncover the identities of the signaling molecules downstream of LT $\beta \mathrm{r}$ in FRCs, differentiating between members of the $\mathrm{NF}-\kappa \mathrm{B}$ pathway and the noncanonical NF- $\mathrm{KB}$ pathway $(75,76)$.

Moreover, some of the effects we observed using LT $\beta r$-Ig could be due to its effects on HEVs (75), and the potential impact of LT $\beta r$-Ig on the formation of tertiary lymphoid tissue (TLT) in the inflamed organ should be investigated. Mii et al. have shown that old mice can develop TLT in the kidneys following IRI, but interestingly young mice lack this capacity (77).

Finally, exogenous FRCs were found to traffic to the KLN during IRI. Administration of FRCs led to the restoration of the microarchitecture within the KLN and significantly ameliorated the development of renal fibrosis following repetitive IRI. Similarly, others have shown that administration of FRCs improved inflammatory responses and survival significantly in a murine model of sepsis (45).

In summary, IRI of the kidney results in activation of the FRCs within the KLN. Depletion or inhibition of FRCs in the acute phase following renal IRI results in the suppression of renal injury. IRI ${ }^{\text {(rep })}$ is associated with the disruption of the organized structural framework within the KLN and impaired renal recovery. Chronic blockade of the LT $\alpha /$ LT $\beta r$ pathway also results in fibrosis of the KLN and kidney. Finally, our findings highlight the considerable potential of FRCs as a cellular therapy to control inflammation and promote renal repair following IRI, one of the most common causes of progressive renal fibrosis.

\section{Methods}

Reagents and antibodies. Tissue culture reagents (PBS, DMEM, Basal Media Eagle, FBS, and penicillin/streptomycin) were purchased from Gibco (a division of ThermoFisher Scientific). Paraformaldehyde (20\%) was purchased from Electron Microscopy Sciences. DT was purchased from List Biological Laboratories, ketamine from Zoetis, and xylazine from Santa Cruz Biotechnology. Triton X-100 was from Acros Organics, phorbol 12-mystirate 13-acetate (PMA) from Sigma-Aldrich, GolgiStop protein transport inhibitor from BD Biosciences, collagenase P from Roche Diagnostics, dispase II from Sigma-Aldrich, DNAse I from Roche Diagnostics, and Dulbecco's PBS from Mediatech. Prolong-Gold mounting media was from Invitrogen.

Antibodies utilized in these studies include Cy3-conjugated anti- $\alpha$ SMA (Sigma-Aldrich, catalog C6198; 1:500), rabbit anti-FN (Abcam, catalog ab23750; 1:500), rat anti-ERTR7 (Santa Cruz Biotechnology, catalog sc73355), rat anti-F4/80 (Abcam, catalog ab6640; 1:500), FITC-conjugated anti-Lotus tetragonolobus lectin (anti-LTL) (Vector Labs, catalog FL-1321; 1:1,000), rabbit anti-Ki67 (Vector Labs, catalog VP-RM04; 1:500), APC-conjugated anti-PDPN (BioLegend, catalog 127409), anti-PDPN (R\&D Systems, catalog AF3244; 1:200), anti-Lrp2/Megalin (Abcam, catalog ab76969; 1:100), FITC-conjugated antiHEV (eBioscience, catalog 53-6036-82), FITC-conjugated anti-Lyve1 (R\&D Systems, catalog FAB2125G), anti-p16 ${ }^{\text {INK4A }}$ (Abcam, catalog ab189034), anti-Coll I (Abcam, catalog ab34710), anti-CD4-Brilliant Violet 510 (eBioscience, catalog 100559) or anti-CD4-PE (eBioscience, catalog 100408), anti-CD44-PE (BioLegend, catalog 103023), and anti-IFN- $\gamma$-APC (BioLegend, catalog 505810). Secondary antibodies were either FITC- or Cy3-conjugated (Jackson ImmunoResearch).

Mice. C57BL/6, B6.129S7-Rag1 ${ }^{\mathrm{tm} 1 \mathrm{Mom} / \mathrm{J}}$ (Rag1--), B6.129S2-Lta ${ }^{\mathrm{tm} 1 \mathrm{Dch} / \mathrm{J}}\left(\mathrm{LT}^{-/-}\right)$, and C57BL/6-Gt (ROSA)26Sor ${ }^{\mathrm{tm} 1}$ (HBEGF)Awai /J (C57BL/6-iDTR) were purchased from Jackson Laboratories. CCL19Cre [Tg (Cc119-cre)489Biat] mice were a gift from Shannon Turley at Genentech, South San Francisco, California, USA. C57BL/6-iDTR mice were backcrossed with CCL19Cre mice and CCL19 ${ }^{\text {Cre }} \times$ iDTR mice were generated. Offspring were genotyped by PCR per the protocol from Jackson Laboratories.

Animal surgery. For the IRI experiments, mice were anesthetized with a mix of ketamine (80-100 mg/ $\mathrm{kg}$ ) and xylazine (5-10 mg/kg) by i.p. injection. Kidneys were exposed through flank incisions. Ischemia was induced by clamping of renal pedicles with nontraumatic microaneurysm clamps (Roboz Surgical Instruments Co.) for either 20 minutes in bilateral IRI or 30 minutes in unilateral IRI. Body temperature was maintained at $36.5^{\circ} \mathrm{C}-37.3^{\circ} \mathrm{C}$. After the clamps were removed, reperfusion of the kidneys was confirmed visually. Blood was taken from the axillary artery at the time of sacrifice. BUN levels were measured using the Infinity Urea assay (ThermoFisher Scientific), according to the manufacturer's instructions.

FRC depletion using DT. CCL19 ${ }^{\text {Cre }} \times$ iDTR mice were injected with 100 ng of DT i.p. on day -2 , day -1 , and day 0 prior to IRI.

LT $\beta$-Ig treatment. LT $\beta r$-Ig and isotype control ragweed-specific mouse IgG2a antibody were gifts from Genentech. C57BL/6 mice were treated with either control IgG $(10 \mathrm{mg} / \mathrm{kg})$ or LT $\beta \mathrm{r}-\mathrm{Ig}(10 \mathrm{mg} / \mathrm{kg})$ every 2 days, starting 4 days prior to IRI. The treatment was continued 3 times per week until the mice were sacrificed. 
Histological studies. Kidneys and KLNs were fixed with 4\% paraformaldehyde for 2 hours on ice, incubated in $30 \%$ (vol/vol) sucrose at $4^{\circ} \mathrm{C}$ overnight, and embedded in optimum cutting temperature compound (Sakura FineTek). For paraffin sections, tissues were fixed with 10\% (vol/vol) formalin, embedded in paraffin, and 5 - $\mu \mathrm{m}$ sections were cut.

For immunostaining of kidney and KLN tissue, frozen sections were cut at $7-\mu \mathrm{m}$ thickness and mounted on Superfrost slides (ThermoFisher Scientific). Sections were washed with PBS and permeabilized with $0.1 \%$ Triton X-100 in PBS. Samples were blocked with 5\% (vol/vol) normal goat serum in PBS and incubated with primary antibodies. They were then washed and incubated with secondary conjugated antibodies. DAPI mixed in Prolong-Gold mounting media was used as a nuclear counterstain. Images were obtained by confocal (Nikon C1 Eclipse) or standard (Nikon Eclipse 90i) microscopy. All images were automatically processed using ImageJ (NIH) and split into RGB channels. Auto threshold was used to convert intensity values of the immunofluorescent stain into numeric data. In tissues stained with $\mathrm{H} \& \mathrm{E}$, quantification of kidney cortex thickness was performed in a scanned whole kidney section, using NIS Elements (Nikon).

Real-time PCR experiments. Tissue or cell pellets were harvested and immediately snap frozen in liquid nitrogen. RNA from tissues was extracted per the manufacturer's instructions, using the RNeasy Mini Kit (Qiagen), and $600 \mathrm{ng}$ of total RNA was reverse transcribed with iScript (Bio-Rad). Quantitative PCR was carried out with iQ-SYBR Green supermix (Bio-Rad) and the Bio-Rad CFX96 Real-Time System with the $\mathrm{C} 1000$ Touch Thermal Cycler, and all the reactions were done in duplicate. Cycling conditions were $95^{\circ} \mathrm{C}$ for 3 minutes, then 40 cycles of $95^{\circ} \mathrm{C}$ for 15 seconds, $60^{\circ} \mathrm{C}$ for 1 minute, followed by 1 cycle of $95^{\circ} \mathrm{C}$ for 10 seconds. Data were analyzed using the $2-\Delta \Delta \mathrm{Ct}$ method. The following primers were used: GAPDH forward 5'-AGCCACATCGCTCAGACAC-3' and reverse 5'-GCCCAATACGACCAAATCC-3'; aSMA forward 5'-CTGACAGAGGCACCACTGAA-3' and reverse 5'-CATCTCCAGAGTCCAGCACA-3'; Col1 $\alpha 1$ forward 5'-TGACTGGAAGAGCGGAGAGT-3' and reverse 5'-GTTCGGGCTGATGTACCAGT-3'; IL-6 forward 5'-CTTCACAAGTCGG AGGCTTAAT-3' and reverse 5'-GCAAGTG CATCATCGTTGTTC-3'; TNF- $\alpha$ forward 5'-CTATGTCTCAGCCTCTTCTCATTC-3' and reverse 5'-GAGGCCATTTGGGAACTTCT-3'; IFN- $\gamma$ forward 5'-TGAACGCTACACACTGCATCTTGG-3' and reverse 5'-CGACTCCTTTTCCGCTTCCTGAG-3'; LT $\beta r$ forward 5'-TGGTGCTCATCССТАССТTCA-3' and reverse 5'-TTCTCTCTATCСТCTCCCCCAG-3'; IL-1 $\beta$ forward 5'-CCTTCCAGGATGAGGACATGA-3' and reverse 5'-AACGTCACACACCAGCAGGTT-3'; F4/80 forward 5'-CCCCAGTGTCCTTACAGAGTG-3' and reverse 5'-GTGCCCAGAGTGGATGTCT-3'; CCL19 forward 5'-TGTGTTCACCACACTAAGGGG-3' and reverse 5'-CCTTTGTTCTTGGCAGAAGACT-3'; PDPN forward 5'-CTCAAGCTTCAAGATGTGGACCGTGCCAGT-3' and reverse 5'-GAGGAATTCGGGCGAGAACCTTCCAGAAAT-3'; FN forward 5'-CGAGGTGACAGAGACCACAA-3' and reverse 5'-CTGGAGTCAAGCCAGACACA-3'.

Flow cytometry. KLNs were placed in $600 \mu \mathrm{l}$ of a digestion mix containing collagenase $\mathrm{P}(0.2 \mathrm{mg} / \mathrm{ml})$, dispase II $(0.8 \mathrm{mg} / \mathrm{ml})$, and DNAse I $(0.1 \mathrm{mg} / \mathrm{ml})$ dissolved in Dulbecco's PBS. After multiple rounds of digestion, the KLNs were dissolved entirely. Kidneys were minced, digested in collagenase P $(0.25 \mathrm{mg} / \mathrm{ml})$ for 30 minutes at $37^{\circ} \mathrm{C}$, and filtered through a $70-\mu \mathrm{m}$ cell strainer. The pooled digested cells were centrifuged, resuspended in DMEM, and counted manually utilizing $0.4 \%$ trypan blue (ThermoFisher Scientific) to exclude dead cells. The cell suspension was placed in 96-well round-bottom plates for intracellular cytokine staining and for surface staining.

These cells were washed and stained according to standard protocols, and flow cytometry was performed via BD FACSCanto II flow cytometer (BD Biosciences). Results were analyzed using FlowJo software (FlowJo LLC).

Electron microscopy. KLNs were fixed in Karnovsky fixative. Samples were cut into approximately 1.5-mm-thick pieces, subjected to a secondary fixation for 1 hour in $1 \%$ osmium tetroxide $/ 0.3 \%$ potassium ferrocyanide in cacodylate buffer on ice, and stained en bloc for 1 hour with $2 \%$ aqueous uranyl acetate. Samples were cured in an oven at $60^{\circ} \mathrm{C}$ for 48 hours. Sections $(70 \mathrm{~nm})$ were then taken and imaged on an FE-SEM (Zeiss Crossbeam 540) using the aSTEM detector.

FRC isolation and expansion. Murine LNs were harvested, digested with $3 \mathrm{ml}$ of enzyme mix containing $0.2 \mathrm{mg} / \mathrm{ml}$ collagenase $\mathrm{P}, 0.1 \mathrm{mg} / \mathrm{ml} \mathrm{DNase} \mathrm{I}$, and $0.8 \mathrm{mg} / \mathrm{ml}$ dispase II, and incubated at $37^{\circ} \mathrm{C}$ for $15 \mathrm{~min}$ utes. LNs were agitated, and the supernatant was collected. The enzyme mix was replaced, and the above steps were repeated until all of the LNs were completely digested. The single-cell suspensions were cultured 
in complete DMEM containing 10\% FBS, 1\% penicillin/streptomycin, and 1\% L-glutamine. The complete DMEM was replaced after 48 hours, and FRCs were allowed to expand for 3 to 4 passages before use. Cells were washed twice with PBS and harvested using 0.25\% trypsin-EDTA (Gibco).

Podocytes. Podocytes were a gift from Anna Greka (Brigham and Women's Hospital).

Senescence-associated $\beta$-galactosidase staining. FRCs were seeded on glass coverslips using 6-well plates. The cells were allowed to attach to coverslips 1 day prior to staining. Then, they were fixed and stained according to the manufacturer's protocol (Senescence $\beta$-Galactosidase staining kit, Cell Signaling Technology, catalog 9860).

Adoptive transfer of $T$ cells. T cells were isolated from splenocytes of either WT mice or $\mathrm{LT}^{-/-}$mice by positive selection using magnetic beads (Pan T Cell Isolation Kit, Miltenyi Biotec). Cells $\left(2.0 \times 10^{7}\right)$ were injected into Rag1 $1^{-/-}$mice 1 day prior to bilateral IRI. Mice were sacrificed 2 days following IRI, and changes in the KLN and kidney were assessed.

FRC labeling and injection into mice. Poly (lactic co-glycolic acid) (PLGA, 50:50 LA/GA [w/w]) NPs were fabricated and loaded with IR $800 \mathrm{CW}$, a near-infrared fluorescent dye, using a nanoprecipitation and solvent evaporation method. The average diameter of IR800-NPs was $60 \mathrm{~nm}$, as measured by dynamic light scattering and transmission electron microscopy. Next, FRCs were incubated with IR800-NPs at $37^{\circ} \mathrm{C}$ for 1 hour and washed via centrifugation. IR800-NP-labeled FRCs (150,000 cells/mouse) were injected intravenously into mice. Kidneys and KLNs were harvested 16 hours after injection and imaged by a UVP iBox Explorer imaging microscope.

Statistics. Data are presented as mean \pm SEM. Statistical analysis was performed using the unpaired 2-tailed Student's $t$ test to determine differences between 2 groups and analysis of variance to compare data among groups. $P$ values of less than 0.05 were considered statistically significant. Each experiment was repeated at least twice with similar results.

Study approval. All experiments were performed according to the animal experimental guidelines upon the approval of the Institutional Animal Care and Use Committee at Brigham and Women's Hospital and Harvard Medical School (Boston, MA).

Data availability. All data generated or analyzed during this study are available from the corresponding author on reasonable request.

\section{Author contributions}

OHM designed and performed experiments, analyzed and interpreted data, and drafted the manuscript. MU performed microsurgery, analyzed and interpreted data, and drafted the manuscript. VK, ZS, NB, BB, LJ, and OAY performed experiments and immunohistochemistry and analyzed data. IG, SBL, JLG, PF, PTS, JSB, and MMM helped with the study design and data analysis, and critically revised the manuscript. RA designed the study, interpreted all data, and critically revised and finalized the manuscript.

\section{Acknowledgments}

This work is supported by the National Institute of Allergy and Infectious Diseases of the NIH under award numbers RO1AI126595 and R56AI123370 (to R. Abdi), a fellowship award from Mallinckrodt Pharmaceuticals (to O.H. Maarouf), and American Heart Association Fellow-to-Faculty Grant Number 14FTF19620001 (to M.M. McGrath). We acknowledge the UAB-UCSD O'Brien Core Center for Acute Kidney Injury Research (NIH P30-DK079337) and Craig Brooks for technical assistance.

Address correspondence to: Reza Abdi, Transplant Research Center, Brigham and Women's Hospital, 221 Longwood Avenue, Boston, Massachusetts 02115, USA. Phone: 617.732.5259; Email: rabdi@rics.bwh. harvard.edu.

1. Brown JR, Rezaee ME, Marshall EJ, Matheny ME. Hospital mortality in the United States following acute kidney injury. Biomed Res Int. 2016;2016:4278579.

2. Wang HE, Muntner P, Chertow GM, Warnock DG. Acute kidney injury and mortality in hospitalized patients. Am J Nephrol. 2012;35(4):349-355.

3. Munshi R, Hsu C, Himmelfarb J. Advances in understanding ischemic acute kidney injury. BMC Med. 2011;9:11.

4. Case J, Khan S, Khalid R, Khan A. Epidemiology of acute kidney injury in the intensive care unit. Crit Care Res Pract. 2013;2013:479730.

5. Schrier RW, Wang W, Poole B, Mitra A. Acute renal failure: definitions, diagnosis, pathogenesis, and therapy. J Clin Invest. 2004;114(1):5-14 
6. Uchino S, et al. Acute renal failure in critically ill patients: a multinational, multicenter study. JAMA. 2005;294(7):813-818.

7. Ishani A, et al. Acute kidney injury increases risk of ESRD among elderly. J Am Soc Nephrol. 2009;20(1):223-228.

8. Bonventre JV, Yang L. Cellular pathophysiology of ischemic acute kidney injury. J Clin Invest. 2011;121(11):4210-4221.

9. Jang HR, Rabb H. Immune cells in experimental acute kidney injury. Nat Rev Nephrol. 2015;11(2):88-101.

10. Itano AA, Jenkins MK. Antigen presentation to naive CD4 T cells in the lymph node. Nat Immunol. 2003;4(8):733-739.

11. von Andrian UH, Mempel TR. Homing and cellular traffic in lymph nodes. Nat Rev Immunol. 2003;3(11):867-878.

12. Ruddle NH, Akirav EM. Secondary lymphoid organs: responding to genetic and environmental cues in ontogeny and the immune response. J Immunol. 2009;183(4):2205-2212.

13. Malhotra D, Fletcher AL, Turley SJ. Stromal and hematopoietic cells in secondary lymphoid organs: partners in immunity. Immunol Rev. 2013;251(1):160-176.

14. Fletcher AL, et al. Reproducible isolation of lymph node stromal cells reveals site-dependent differences in fibroblastic reticular cells. Front Immunol. 2011;2:35.

15. Cremasco V, et al. B cell homeostasis and follicle confines are governed by fibroblastic reticular cells. Nat Immunol. 2014;15(10):973-981.

16. Severino P, et al. Human lymph node-derived fibroblastic and double-negative reticular cells alter their chemokines and cytokines expression profile following inflammatory stimuli. Front Immunol. 2017;8:141.

17. Hinz B, Phan SH, Thannickal VJ, Galli A, Bochaton-Piallat ML, Gabbiani G. The myofibroblast: one function, multiple origins. Am J Pathol. 2007;170(6):1807-1816.

18. Miyasaka M, Tanaka T. Lymphocyte trafficking across high endothelial venules: dogmas and enigmas. Nat Rev Immunol. 2004;4(5):360-370.

19. Stein JV, Nombela-Arrieta C. Chemokine control of lymphocyte trafficking: a general overview. Immunology. 2005;116(1):1-12.

20. Denton AE, Roberts EW, Linterman MA, Fearon DT. Fibroblastic reticular cells of the lymph node are required for retention of resting but not activated CD8 ${ }^{+}$T cells. Proc Natl Acad Sci USA. 2014;111(33):12139-12144.

21. Astarita JL, Acton SE, Turley SJ. Podoplanin: emerging functions in development, the immune system, and cancer. Front Immunol. $2012 ; 3: 283$.

22. Acton SE, et al. Dendritic cells control fibroblastic reticular network tension and lymph node expansion. Nature. 2014;514(7523):498-502.

23. Fletcher AL, Acton SE, Knoblich K. Lymph node fibroblastic reticular cells in health and disease. Nat Rev Immunol. 2015;15(6):350-361.

24. Zeng M, et al. Lymphoid tissue damage in HIV-1 infection depletes naïve T cells and limits T cell reconstitution after antiretroviral therapy. PLoS Pathog. 2012;8(1):e1002437.

25. Estes JD, et al. Antifibrotic therapy in simian immunodeficiency virus infection preserves $\mathrm{CD}^{+}{ }^{+} \mathrm{T}$-cell populations and improves immune reconstitution with antiretroviral therapy. J Infect Dis. 2015;211(5):744-754.

26. Schacker TW, et al. Collagen deposition in HIV-1 infected lymphatic tissues and T cell homeostasis. J Clin Invest. 2002;110(8):1133-1139.

27. Rabb H, et al. Inflammation in AKI: Current understanding, key questions, and knowledge gaps. J Am Soc Nephrol. 2016;27(2):371-379.

28. Childs BG, Baker DJ, Kirkland JL, Campisi J, van Deursen JM. Senescence and apoptosis: dueling or complementary cell fates? EMBO Rep. 2014;15(11):1139-1153.

29. Childs BG, Durik M, Baker DJ, van Deursen JM. Cellular senescence in aging and age-related disease: from mechanisms to therapy. Nat Med. 2015;21(12):1424-1435.

30. Banerjee I, Yekkala K, Borg TK, Baudino TA. Dynamic interactions between myocytes, fibroblasts, and extracellular matrix. Ann N Y Acad Sci. 2006;1080:76-84.

31. Moore-Morris T, et al. Resident fibroblast lineages mediate pressure overload-induced cardiac fibrosis. J Clin Invest. 2014;124(7):2921-2934.

32. Karin D, Koyama Y, Brenner D, Kisseleva T. The characteristics of activated portal fibroblasts/myofibroblasts in liver fibrosis Differentiation. 2016;92(3):84-92.

33. Hundae A, McCullough PA. Cardiac and renal fibrosis in chronic cardiorenal syndromes. Nephron Clin Pract. 2014;127(14):106-112.

34. Frantz C, Stewart KM, Weaver VM. The extracellular matrix at a glance. J Cell Sci. 2010;123(Pt 24):4195-4200.

35. Cotran RS. The delayed and prolonged vascular leakage in inflammation. II. An electron microscopic study of the vascular response after thermal injury. Am J Pathol. 1965;46:589-620.

36. Dunn WA. Studies on the mechanisms of autophagy: maturation of the autophagic vacuole. J Cell Biol. 1990;110(6):1935-1945.

37. Abe J, et al. Lymph node stromal cells negatively regulate antigen-specific CD4+ T cell responses. J Immunol. 2014;193(4):1636-1644.

38. Dubey LK, et al. Lymphotoxin-dependent B cell-FRC crosstalk promotes de novo follicle formation and antibody production following intestinal helminth infection. Cell Rep. 2016;15(7):1527-1541.

39. Yang CY, et al. Trapping of naive lymphocytes triggers rapid growth and remodeling of the fibroblast network in reactive murine lymph nodes. Proc Natl Acad Sci USA. 2014;111(1):E109-E118.

40. Baekkevold ES, et al. The CCR7 ligand elc (CCL19) is transcytosed in high endothelial venules and mediates T cell recruitment. J Exp Med. 2001;193(9):1105-1112.

41. Chai Q, et al. Maturation of lymph node fibroblastic reticular cells from myofibroblastic precursors is critical for antiviral immunity. Immunity. 2013;38 (5):1013-1024.

42. Linfert D, Chowdhry T, Rabb H. Lymphocytes and ischemia-reperfusion injury. Transplant Rev (Orlando). 2009;23(1):1-10.

43. Förster R, Moschovakis GL. Orchestrating the organizers: lymphotoxin- $\beta$ receptor conducts fibroblastic reticular cell maturation. Immunity. 2013;38(5):851-853.

44. Ruddle NH. Lymphotoxin and TNF: how it all began-a tribute to the travelers. Cytokine Growth Factor Rev. 2014;25(2):83-89.

45. Fletcher AL, et al. Lymph node fibroblastic reticular cell transplants show robust therapeutic efficacy in high-mortality murine sepsis. Sci Transl Med. 2014;6(249):249ra109. 
46. Bajénoff M, et al. Stromal cell networks regulate lymphocyte entry, migration, and territoriality in lymph nodes. Immunity. 2006;25(6):989-1001.

47. Link A, et al. Fibroblastic reticular cells in lymph nodes regulate the homeostasis of naive T cells. Nat Immunol. 2007;8(11):1255-1265.

48. Schumann K, et al. Immobilized chemokine fields and soluble chemokine gradients cooperatively shape migration patterns of dendritic cells. Immunity. 2010;32(5):703-713.

49. Scandella E, et al. Restoration of lymphoid organ integrity through the interaction of lymphoid tissue-inducer cells with stroma of the T cell zone. Nat Immunol. 2008;9(6):667-675.

50. Fine LG, Bandyopadhay D, Norman JT. Is there a common mechanism for the progression of different types of renal diseases other than proteinuria? Towards the unifying theme of chronic hypoxia. Kidney Int Suppl. 2000;75:S22-S26.

51. Zeng M, et al. Cumulative mechanisms of lymphoid tissue fibrosis and T cell depletion in HIV-1 and SIV infections. JClin Invest. 2011;121(3):998-1008.

52. O'Brien J, et al. Alternatively activated macrophages and collagen remodeling characterize the postpartum involuting mammary gland across species. Am J Pathol. 2010;176(3):1241-1255

53. Weathington NM, et al. A novel peptide CXCR ligand derived from extracellular matrix degradation during airway inflammation. Nat Med. 2006;12(3):317-323.

54. Herzog BH, et al. Podoplanin maintains high endothelial venule integrity by interacting with platelet CLEC-2. Nature. 2013;502(7469):105-109.

55. Wight TN, Potter-Perigo S. The extracellular matrix: an active or passive player in fibrosis? Am J Physiol Gastrointest Liver Physiol. 2011;301(6):G950-G955.

56. Li S, Van Den Diepstraten C, D'Souza SJ, Chan BM, Pickering JG. Vascular smooth muscle cells orchestrate the assembly of type I collagen via alpha2beta1 integrin, RhoA, and fibronectin polymerization. Am J Pathol. 2003;163(3):1045-1056

57. Berk BC, Fujiwara K, Lehoux S. ECM remodeling in hypertensive heart disease. J Clin Invest. 2007;117(3):568-575

58. Astarita JL, et al. The CLEC-2-podoplanin axis controls the contractility of fibroblastic reticular cells and lymph node microarchitecture. Nat Immunol. 2015;16(1):75-84.

59. Wang D, Jang DJ. Protein kinase CK2 regulates cytoskeletal reorganization during ionizing radiation-induced senescence of human mesenchymal stem cells. Cancer Res. 2009;69(20):8200-8207.

60. Turinetto V, Vitale E, Giachino C. Senescence in human mesenchymal stem cells: Functional changes and implications in stem cell-based therapy. Int J Mol Sci. 2016;17(7):1164.

61. Freund A, Orjalo AV, Desprez PY, Campisi J. Inflammatory networks during cellular senescence: causes and consequences. Trends Mol Med. 2010;16(5):238-246.

62. Kuilman T, et al. Oncogene-induced senescence relayed by an interleukin-dependent inflammatory network. Cell. 2008;133(6):1019-1031.

63. Sudhamsu J, Yin J, Chiang EY, Starovasnik MA, Grogan JL, Hymowitz SG. Dimerization of LT $\beta$ R by LT $\alpha 1 \beta 2$ is necessary and sufficient for signal transduction. Proc Natl Acad Sci USA. 2013;110(49):19896-19901.

64. Cheng HW, et al. CCL19-producing fibroblastic stromal cells restrain lung carcinoma growth by promoting local antitumor T-cell responses [published online ahead of print January 31, 2018]. J Allergy Clin Immunol. https://doi.org/10.1016/j. jaci.2017.12.998.

65. Rudnicki M, et al. Renal microRNA- and RNA-profiles in progressive chronic kidney disease. Eur J Clin Invest. 2016;46(3):213-226

66. Valiño-Rivas L, Gonzalez-Lafuente L, Sanz AB, Ruiz-Ortega M, Ortiz A, Sanchez-Niño MD. Non-canonical NFкB activation promotes chemokine expression in podocytes. Sci Rep. 2016;6:28857.

67. Mounayar M, et al. PI3k $\alpha$ and STAT1 interplay regulates human mesenchymal stem cell immune polarization. Stem Cells. 2015;33(6):1892-1901.

68. Lukacs-Kornek V, et al. Regulated release of nitric oxide by nonhematopoietic stroma controls expansion of the activated $\mathrm{T}$ cell pool in lymph nodes. Nat Immunol. 2011;12(11):1096-1104.

69. Suenaga F, et al. Loss of lymph node fibroblastic reticular cells and high endothelial cells is associated with humoral immunodeficiency in mouse graft-versus-host disease. J Immunol. 2015;194(1):398-406.

70. Malhotra D, et al. Transcriptional profiling of stroma from inflamed and resting lymph nodes defines immunological hallmarks. Nat Immunol. 2012;13(5):499-510.

71. Dubrot J, et al. Lymph node stromal cells acquire peptide-MHCII complexes from dendritic cells and induce antigen-specific CD4+ T cell tolerance. J Exp Med. 2014;211(6):1153-1166.

72. Seleznik G, et al. The lymphotoxin $\beta$ receptor is a potential therapeutic target in renal inflammation. Kidney Int. 2016;89(1):113-126

73. Nakayama Y, Bromberg JS. Lymphotoxin-beta receptor blockade induces inflammation and fibrosis in tolerized cardiac allografts. Am J Transplant. 2012;12(9):2322-2334.

74. Lee Y, et al. Recruitment and activation of naive T cells in the islets by lymphotoxin beta receptor-dependent tertiary lymphoid structure. Immunity. 2006;25(3):499-509.

75. Browning JL, et al. Lymphotoxin-beta receptor signaling is required for the homeostatic control of HEV differentiation and function. Immunity. 2005;23(5):539-550.

76. Ager A, May MJ. Understanding high endothelial venules: Lessons for cancer immunology. Oncoimmunology. 2015;4(6):e1008791.

77. Sato Y, et al. Heterogeneous fibroblasts underlie age-dependent tertiary lymphoid tissues in the kidney. JCI Insight 2016;1(11):e87680. 